\title{
A Simple Approach for Monitoring Business Service Time Variation
}

\author{
Su-Fen Yang ${ }^{1}$ and Barry C. Arnold ${ }^{2}$ \\ ${ }^{1}$ Department of Statistics, National Chengchi University, Taipei 116, Taiwan \\ ${ }^{2}$ Department of Statistics, University of California, Riverside, CA 92521, USA \\ Correspondence should be addressed to Su-Fen Yang; yang@nccu.edu.tw
}

Received 31 August 2013; Accepted 8 April 2014; Published 7 May 2014

Academic Editors: V. Bagdonavicius and Y. Zhang

Copyright (C) 2014 S.-F. Yang and B. C. Arnold. This is an open access article distributed under the Creative Commons Attribution License, which permits unrestricted use, distribution, and reproduction in any medium, provided the original work is properly cited.

\begin{abstract}
Control charts are effective tools for signal detection in both manufacturing processes and service processes. Much of the data in service industries comes from processes having nonnormal or unknown distributions. The commonly used Shewhart variable control charts, which depend heavily on the normality assumption, are not appropriately used here. In this paper, we propose a new asymmetric EWMA variance chart (EWMA-AV chart) and an asymmetric EWMA mean chart (EWMA-AM chart) based on two simple statistics to monitor process variance and mean shifts simultaneously. Further, we explore the sampling properties of the new monitoring statistics and calculate the average run lengths when using both the EWMA-AV chart and the EWMA-AM chart. The performance of the EWMA-AV and EWMA-AM charts and that of some existing variance and mean charts are compared. A numerical example involving nonnormal service times from the service system of a bank branch in Taiwan is used to illustrate the applications of the EWMA-AV and EWMA-AM charts and to compare them with the existing variance (or standard deviation) and mean charts. The proposed EWMA-AV chart and EWMA-AM charts show superior detection performance compared to the existing variance and mean charts. The EWMA-AV chart and EWMA-AM chart are thus recommended.
\end{abstract}

\section{Introduction}

Control charts are commonly used tools in process signal detection to improve the quality of manufacturing processes and service processes. In the past few years, more and more statistical process control techniques have been applied to the service industry, and control charts are also becoming an effective tool in improving service quality. There have been a few studies in this area, like those of MacCarthy and Wasusri [1], Tsung et al. [2], and Ning et al. [3]. Much service process data come from processes with variables having nonnormal or unknown distributions so the commonly used Shewhart variables control charts, which depend on a normality assumption, are not suitable. Hence the following question arises: "how to monitor the process with nonnormal or unknown distribution data?" Some research has been done to deal with such a situation; see, for example, Ferrell [4]; Bakir and Reynolds [5]; Amin et al. [6]; Chakraborti et al. [7]; Altukife [8, 9]; Bakir [10]; Chakraborti and Eryilmaz [11];
Chakraborti and Graham [12]; Chakraborti and van der Wiel [13]; Li et al. [14]; and Zou and Tsung [15]. Little research has been done to deal with process variability monitoring; see, for example, Das and Bhattacharya [16].

A major drawback of the previous nonparametric approaches is that they are not easy for practitioners to apply because they are not statisticians and do not quite understand the proper way to implement the schemes. Yang et al. [17] proposed a new sign chart for variables data to monitor the deviation of the process measurement from the target without the assumption of a normal process distribution or a distribution of known form. Yang and Cheng [18] proposed a CUSUM mean chart to monitor small shifts in the process mean. Yang et al. [19] addressed a new mean chart based on a simple statistic to monitor the shifts of the process mean. Their approaches are quite easy to use, and even easier than some of the above published nonparametric approaches. However, the mean charts based on an asymmetric binomial distribution (i.e., when $p \neq 1 / 2$ ) do not exhibit a regular 
in-control run length. Moreover the corresponding out-ofcontrol average run lengths do not uniformly decrease as sample size increases as they should. Further, Yang and Cheng [18], Yang et al. [17], and Yang et al. [19] did not consider a variance chart.

In this paper, we propose an improved asymmetric EWMA mean chart (EWMA-AM chart) and a new asymmetric EWMA variance chart (EWMA-AV chart) for variables data to effectively monitor the process mean and variance simultaneously. The approach is still quite easy to use and has better detection ability than the existing mean and standard deviation charts. The paper is organized as follows. In Section 2, we propose the exponentially weighted moving average (EWMA) chart, EWMA-AM chart, to detect the out-of-control process mean and measure its performance. In Section 3, we discuss the construction of a newly proposed EWMA-AV chart to detect the out-of-control process variance and its performance. In Section 4, we propose to combine the two EWMA charts, EWMA-AM chart and EWMA-AV chart, to detect both the out-of-control mean and variance and measure the performance. In Section 5, we describe the estimates for the unknown process mean and variance. In Section 6, a numerical example of a service system in a bank branch was used to construct the proposed EWMA-AM chart and EWMA-AV chart to monitor the quality of service time and their performance compared with those of existing charts. Section 7 summarizes the findings and provides a recommendation.

\section{The Proposed EWMA-AM Chart}

Assume that a critical quality characteristic, $X$, has a mean $\mu$ and variance $\sigma^{2}$.

Following Yang et al. [17], let $Y=X-\mu$ and $p=P(Y>$ $0)=$ the "Process Proportion." If the process was in control then $p=p_{m 0}$, and if the process was out of control, that is, $\mu$ had shifted; then $p=p_{m 1} \neq p_{m 0}$. If $p_{m 0}$ is not given, it will be estimated using a preliminary data set.

To monitor the process mean, a random sample of size $n_{1}$, $X_{1}, X_{2}, \ldots, X_{n_{1}}$, is taken from $X$. Define

$$
Y_{j}=X_{j}-\mu, \quad I_{j}=\left\{\begin{array}{ll}
1, & \text { if } Y_{j}>0, \\
0, & \text { otherwise, }
\end{array} \quad j=1,2, \ldots, n_{1} .\right.
$$

Let $M_{t}$ be the total number of $Y_{j}>0$ at time $t$; then $M_{t}=$ $\sum_{j=1}^{n_{1}} I_{j}$ would follow a binomial distribution with parameters $\left(n_{1}, p_{m 0}\right)$ for an in-control process.

Based on the distribution of $M$, the $M$ chart could be constructed to monitor the process proportion. Monitoring the process mean shifts is equivalent to monitoring the changes in process proportion.

2.1. The Control Limits of EWMA-AM Chart. To overcome the defects of using the symmetric mean chart and to have superior performance in detecting small shifts in the process mean, a new improved asymmetric EWMA mean chart (EWMA-AM chart) is proposed. The EWMA control charts have been demonstrated to have better performance for detecting small shifts in process parameters when compared with Shewhart-type charts (e.g., see [20]). The new improved asymmetric EWMA mean chart (EWMA-AM chart) has upper control limit $\left(\mathrm{UCL}_{\mathrm{EWMA}_{M}}\right)$, central line $\left(\mathrm{CL}_{\mathrm{EWMA}_{M}}\right)$, and lower control limit ( $\mathrm{LCL}_{\mathrm{EWMA}_{M}}$ ) defined as follows:

$$
\begin{aligned}
& \mathrm{UCL}_{\mathrm{EWMA}_{M}}= n_{1} p_{m 0}+k_{3} \sqrt{\frac{\lambda_{1}}{\left(2-\lambda_{1}\right)} n_{1} p_{m 0}\left(1-p_{m 0}\right)}, \\
& \mathrm{CL}_{\mathrm{EWMA}_{M}}=n_{1} p_{m 0}, \\
& \mathrm{LCL}_{\mathrm{EWMA}_{M}}=n_{1} p_{m 0}-k_{4} \sqrt{\frac{\lambda_{1}}{\left(2-\lambda_{1}\right)} n_{1} p_{m 0}\left(1-p_{m 0}\right)},
\end{aligned}
$$

where $k_{3}$ and $k_{4}$ are appropriately chosen coefficients for the $\mathrm{UCL}_{\mathrm{EWMA}_{M}}$ and $\mathrm{LCL}_{\mathrm{EWMA}_{M}}$, respectively.

If any monitoring statistic EWMA $M_{t}$ exceeds $\mathrm{UCL}_{\mathrm{EWMA}_{M}}$ or if EWMA ${ }_{M_{t}}$ falls below $\mathrm{LCL}_{\mathrm{EWMA}_{M}}$, the process is deemed to exhibit some out-of-mean-control signal.

The monitoring statistic EWMA ${ }_{M_{t}}$ based on the statistic $M$ is defined as follows:

$$
\begin{aligned}
\text { EWMA }_{M_{t}}= & \lambda M_{t}+\left(1-\lambda_{1}\right) \text { EWMA }_{M_{t-1}} \\
& 0<\lambda_{1} \leq 1, \quad t=1,2,3, \ldots
\end{aligned}
$$

Let the starting value, $\mathrm{EWMA}_{M_{0}}$, be the mean of $M$; that is, $\mathrm{EWMA}_{M_{0}}=n_{1} p_{m 0}$ for an in-control process. Hence the mean and variance of $\mathrm{EWMA}_{M_{t}}$ are

$$
\begin{gathered}
E\left(\mathrm{EWMA}_{M_{t}}\right)=n_{1} p_{m 0} \\
\operatorname{Var}\left(\mathrm{EWMA}_{M_{t}}\right)=\frac{\lambda_{1}\left[1-\left(1-\lambda_{1}\right)^{2 t}\right]}{2-\lambda_{1}}\left(n_{1} p_{m 0}\left(1-p_{m 0}\right)\right) .
\end{gathered}
$$

The asymptotic variance of $\mathrm{EWMA}_{M_{t}}$ is

$$
\operatorname{Var}\left(\operatorname{EWMA}_{M_{t}}\right)=\frac{\lambda_{1}}{2-\lambda_{1}}\left(n_{1} p_{m 0}\left(1-p_{m 0}\right)\right) .
$$

To measure the performance of the proposed new EWMA-AM chart, we calculated the average run length (ARL). The in-control ARL, ARL ${ }_{m 0}$, of the EWMA-AM chart depends on the values of $n_{1}, k_{3}, k_{4}, \lambda_{1}$, and $p_{m 0}$. The two parameters, $k_{3}$ and $k_{4}$, for a specified $\lambda$ and $n_{1}=10(1) 30$ are chosen to satisfy a required in-control average run length $\left(\mathrm{ARL}_{m 0}\right) \approx 370$ using a Markov chain approach [21], and the calculation procedure follows that used in Yang [22]. Table 1 shows the values of $k_{3}$ and $k_{4}$ with $\lambda_{1}=0.05$ and $\mathrm{ARL}_{m 0} \approx$ 370 for $n_{1}=10(1) 30$ and $p_{m 0}=0.1(0.1) 0.9$.

2.2. The Out-of-Control Average Run Lengths of the EWMA$A M$ Chart. For the out-of-control process it is assumed that the mean $\mu$ has shifted, and the process proportion has become $p_{m 1}\left(\neq p_{m 0}\right)$. Let $\mathrm{ARL}_{m 1}$ be the out-of-control ARL of the EWMA-AM chart. A small $\mathrm{ARL}_{m 1}$ indicates superior out-of-control detection performance of the control chart. 
TABLE 1: The $k_{3}$ and $k_{4}$ with $\mathrm{ARL}_{m 0} \approx 370$ for various $n_{1}$ and $p_{m 0}$ given $\lambda_{1}=0.05$.

\begin{tabular}{|c|c|c|c|c|c|c|c|c|c|c|c|c|c|c|c|c|c|c|}
\hline \multirow{3}{*}{0} & \multicolumn{18}{|c|}{$p_{m 0}$} \\
\hline & $k_{3}$ & 2.61 & $k_{3}$ & 2.54 & $k_{3}$ & 2.49 & $k_{3}$ & 2.46 & $k_{3}$ & 2.44 & $k_{3}$ & 2.40 & $k_{3}$ & 2.31 & $k_{3}$ & 2.29 & $k_{3}$ & 2.19 \\
\hline & $k_{4}$ & 2.36 & $k_{4}$ & 2.44 & $k_{4}$ & 2.49 & $k_{4}$ & 2.53 & $k_{4}$ & 2.54 & $k_{4}$ & 2.59 & $k_{4}$ & 2.72 & $k_{4}$ & 2.73 & $k_{4}$ & 2.89 \\
\hline \multirow[t]{2}{*}{1} & $k_{3}$ & 2.61 & $k_{3}$ & 2.54 & $k_{3}$ & 2.49 & $k_{3}$ & 2.44 & $k_{3}$ & 2.45 & $k_{3}$ & 2.44 & $k_{3}$ & 2.39 & $k_{3}$ & 2.32 & $k_{3}$ & 2.10 \\
\hline & $k_{4}$ & 2.36 & $k_{4}$ & 2.44 & $k_{4}$ & 2.48 & $k_{4}$ & 2.54 & $k_{4}$ & 2.53 & $k_{4}$ & 2.54 & $k_{4}$ & 2.59 & $k_{4}$ & 2.67 & $k_{4}$ & 3.17 \\
\hline \multirow{2}{*}{12} & $k_{3}$ & 2.60 & $k_{3}$ & 2.53 & $k_{3}$ & 2.49 & $k_{3}$ & 2.45 & $k_{3}$ & 2.45 & $k_{3}$ & 2.42 & $k_{3}$ & 2.40 & $k_{3}$ & 2.33 & $k_{3}$ & 2.35 \\
\hline & $k_{4}$ & 2.36 & $k_{4}$ & 2.44 & $k_{4}$ & 2.48 & $k_{4}$ & 2.53 & $k_{4}$ & 2.54 & $k_{4}$ & 2.57 & $k_{4}$ & 2.57 & $k_{4}$ & 2.65 & $k_{4}$ & 2.62 \\
\hline \multirow{2}{*}{13} & $k_{3}$ & 2.58 & $k_{3}$ & 2.53 & $k_{3}$ & 2.48 & $k_{3}$ & 2.45 & $k_{3}$ & 2.44 & $k_{3}$ & 2.40 & $k_{3}$ & 2.37 & $k_{3}$ & 2.36 & $k_{3}$ & 2.34 \\
\hline & $k_{4}$ & 2.38 & $k_{4}$ & 2.43 & $k_{4}$ & 2.50 & $k_{4}$ & 2.53 & $k_{4}$ & 2.54 & $k_{4}$ & 2.58 & $k_{4}$ & 2.61 & $k_{4}$ & 2.62 & $k_{4}$ & 2.63 \\
\hline \multirow{2}{*}{14} & $k_{3}$ & 2.58 & $k_{3}$ & 2.51 & $k_{3}$ & 2.49 & $k_{3}$ & 2.47 & $k_{3}$ & 2.45 & $k_{3}$ & 2.41 & $k_{3}$ & 2.38 & $k_{3}$ & 2.37 & $k_{3}$ & 2.35 \\
\hline & $k_{4}$ & 2.35 & $k_{4}$ & 2.47 & $k_{4}$ & 2.49 & $k_{4}$ & 2.50 & $k_{4}$ & 2.53 & $k_{4}$ & 2.57 & $k_{4}$ & 2.60 & $k_{4}$ & 2.61 & $k_{4}$ & 2.60 \\
\hline \multirow{2}{*}{15} & $k_{3}$ & 2.57 & $k_{3}$ & 2.51 & $k_{3}$ & 2.49 & $k_{3}$ & 2.47 & $k_{3}$ & 2.41 & $k_{3}$ & 2.39 & $k_{3}$ & 2.36 & $k_{3}$ & 2.43 & $k_{3}$ & 2.41 \\
\hline & $k_{4}$ & 2.37 & $k_{4}$ & 2.47 & $k_{4}$ & 2.49 & $k_{4}$ & 2.51 & $k_{4}$ & 2.58 & $k_{4}$ & 2.60 & $k_{4}$ & 2.64 & $k_{4}$ & 2.55 & $k_{4}$ & 2.53 \\
\hline \multirow{2}{*}{16} & $k_{3}$ & 2.57 & $k_{3}$ & 2.51 & $k_{3}$ & 2.46 & $k_{3}$ & 2.47 & $k_{3}$ & 2.44 & $k_{3}$ & 2.43 & $k_{3}$ & 2.39 & $k_{3}$ & 2.37 & $k_{3}$ & 2.45 \\
\hline & $k_{4}$ & 2.38 & $k_{4}$ & 2.46 & $k_{4}$ & 2.52 & $k_{4}$ & 2.50 & $k_{4}$ & 2.54 & $k_{4}$ & 2.55 & $k_{4}$ & 2.60 & $k_{4}$ & 2.62 & $k_{4}$ & 2.51 \\
\hline \multirow{2}{*}{17} & $k_{3}$ & 2.57 & $k_{3}$ & 2.51 & $k_{3}$ & 2.48 & $k_{3}$ & 2.42 & $k_{3}$ & 2.44 & $k_{3}$ & 2.40 & $k_{3}$ & 2.39 & $k_{3}$ & 2.06 & $k_{3}$ & 2.44 \\
\hline & $k_{4}$ & 2.41 & $k_{4}$ & 2.46 & $k_{4}$ & 2.50 & $k_{4}$ & 2.56 & $k_{4}$ & 2.55 & $k_{4}$ & 2.59 & $k_{4}$ & 59 & $k_{4}$ & .63 & $k_{4}$ & .53 \\
\hline 18 & $k_{3}$ & 2.57 & $k_{3}$ & 2.51 & $k_{3}$ & 2.47 & $k_{3}$ & 2.44 & $k_{3}$ & 2.44 & $k_{3}$ & 2.40 & $k_{3}$ & 2.37 & $k_{3}$ & 2.37 & $k_{3}$ & 2.39 \\
\hline 10 & $k_{4}$ & 2.40 & $k_{4}$ & 2.46 & $k_{4}$ & 2.51 & $k_{4}$ & 2.54 & $k_{4}$ & 2.54 & $k_{4}$ & 2.59 & $k_{4}$ & 2.62 & $k_{4}$ & 2.61 & $k_{4}$ & 2.59 \\
\hline 19 & $k_{3}$ & 2.57 & $k_{3}$ & 2.51 & $k_{3}$ & 2.48 & $k_{3}$ & 2.47 & $k_{3}$ & 2.47 & $k_{3}$ & 2.43 & $k_{3}$ & 2.27 & $k_{3}$ & 2.20 & $k_{3}$ & 2.46 \\
\hline & $k_{4}$ & 2.41 & $k_{4}$ & 2.47 & $k_{4}$ & 2.50 & $k_{4}$ & 2.51 & $k_{4}$ & 2.51 & $k_{4}$ & 2.55 & $k_{4}$ & 2.79 & $k_{4}$ & 2.94 & $k_{4}$ & 2.51 \\
\hline 20 & $k_{3}$ & 2.55 & $k_{3}$ & 2.51 & $k_{3}$ & 2.46 & $k_{3}$ & 2.47 & $k_{3}$ & 2 & $k_{3}$ & 42 & $k_{3}$ & 42 & $k_{3}$ & .42 & $k_{3}$ & 2.5 \\
\hline & $k_{4}$ & 2.42 & $k_{4}$ & 2.46 & $k_{4}$ & 2.51 & $k_{4}$ & 2.50 & $k_{4}$ & 2.55 & $k_{4}$ & 2.55 & $k_{4}$ & 2.56 & $k_{4}$ & 2.56 & $k_{4}$ & 2.48 \\
\hline 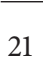 & $k_{3}$ & 2.55 & $k_{3}$ & 2.49 & $k_{3}$ & 2.47 & $k_{3}$ & 2.46 & $k_{3}$ & 2.45 & $k_{3}$ & 2.42 & $k_{3}$ & 2.50 & $k_{3}$ & 2.40 & $k_{3}$ & 2.5 \\
\hline & $k_{4}$ & 2.42 & $k_{4}$ & 2.48 & $k_{4}$ & 2.51 & $k_{4}$ & 2.52 & $k_{4}$ & 2.54 & $k_{4}$ & 2.56 & $k_{4}$ & 2.48 & $k_{4}$ & 2.57 & $k_{4}$ & 2.48 \\
\hline 22 & $k_{3}$ & 2.55 & $k_{3}$ & 2.51 & $k_{3}$ & 2.48 & $k_{3}$ & 2.43 & $k_{3}$ & 2.42 & $k_{3}$ & 2.36 & $k_{3}$ & 2.29 & $k_{3}$ & 2.42 & $k_{3}$ & 2.47 \\
\hline & $k_{4}$ & 2.42 & $k_{4}$ & 2.47 & $k_{4}$ & 2.51 & $k_{4}$ & 2.55 & $k_{4}$ & 2.57 & $k_{4}$ & 2.64 & $k_{4}$ & 2.76 & $k_{4}$ & 2.57 & $k_{4}$ & 2.5 \\
\hline 23 & $k_{3}$ & 2.56 & $k_{3}$ & 2.50 & $k_{3}$ & 2.49 & $k_{3}$ & 2.46 & $k_{3}$ & 2.45 & $k_{3}$ & 2.47 & $k_{3}$ & 2.32 & $k_{3}$ & 2.47 & $k_{3}$ & 2.6 \\
\hline 23 & $k_{4}$ & 2.41 & $k_{4}$ & 2.48 & $k_{4}$ & 2.47 & $k_{4}$ & 2.52 & $k_{4}$ & 2.53 & $k_{4}$ & 2.50 & $k_{4}$ & 2.71 & $k_{4}$ & 2.51 & $k_{4}$ & 2.4 \\
\hline 24 & $k_{3}$ & 2.54 & $k_{3}$ & 2.50 & $k_{3}$ & 2.47 & $k_{3}$ & 2.45 & $k_{3}$ & 2.44 & $k_{3}$ & 2.34 & $k_{3}$ & 2.43 & $k_{3}$ & 2.43 & $k_{3}$ & 2.52 \\
\hline & $k_{4}$ & 2.42 & $k_{4}$ & 2.48 & $k_{4}$ & 2.50 & $k_{4}$ & 2.53 & $k_{4}$ & 2.54 & $k_{4}$ & 2.69 & $k_{4}$ & 2.55 & $k_{4}$ & 2.55 & $k_{4}$ & 2.45 \\
\hline 25 & $k_{3}$ & 2.56 & $k_{3}$ & 2.50 & $k_{3}$ & 2.48 & $k_{3}$ & 2.45 & $k_{3}$ & 2.46 & $k_{3}$ & 2.35 & $k_{3}$ & 2.43 & $k_{3}$ & 2.37 & $k_{3}$ & 2.28 \\
\hline 20 & $k_{4}$ & 2.41 & $k_{4}$ & 2.48 & $k_{4}$ & 2.50 & $k_{4}$ & 2.52 & $k_{4}$ & 2.53 & $k_{4}$ & 2.68 & $k_{4}$ & 2.56 & $k_{4}$ & 2.62 & $k_{4}$ & 2.74 \\
\hline 26 & $k_{3}$ & 2.56 & $k_{3}$ & 2.49 & $k_{3}$ & 2.47 & $k_{3}$ & 2.45 & $k_{3}$ & 2.45 & $k_{3}$ & 2.42 & $k_{3}$ & 2.44 & $k_{3}$ & 2.43 & $k_{3}$ & 2.43 \\
\hline & $k_{4}$ & 2.41 & $k_{4}$ & 2.49 & $k_{4}$ & 2.50 & $k_{4}$ & 2.54 & $k_{4}$ & 2.53 & $k_{4}$ & 2.56 & $k_{4}$ & 2.55 & $k_{4}$ & 2.54 & $k_{4}$ & 2.53 \\
\hline 27 & $k_{3}$ & 2.54 & $k_{3}$ & 2.50 & $k_{3}$ & 2.47 & $k_{3}$ & 2.46 & $k_{3}$ & 2.49 & $k_{3}$ & 2.45 & $k_{3}$ & 2.42 & $k_{3}$ & 2.40 & $k_{3}$ & 2.37 \\
\hline & $k_{4}$ & 2.42 & $k_{4}$ & 2.48 & $k_{4}$ & 2.51 & $k_{4}$ & 2.52 & $k_{4}$ & 2.49 & $k_{4}$ & 2.53 & $k_{4}$ & 2.56 & $k_{4}$ & 2.58 & $k_{4}$ & 2.60 \\
\hline 28 & $k_{3}$ & 2.55 & $k_{3}$ & 2.49 & $k_{3}$ & 2.47 & $k_{3}$ & 2.46 & $k_{3}$ & 2.35 & $k_{3}$ & 2.40 & $k_{3}$ & 2.40 & $k_{3}$ & 2.35 & $k_{3}$ & 2.30 \\
\hline 28 & $k_{4}$ & 2.42 & $k_{4}$ & 2.49 & $k_{4}$ & 2.51 & $k_{4}$ & 2.52 & $k_{4}$ & 2.67 & $k_{4}$ & 2.59 & $k_{4}$ & 2.58 & $k_{4}$ & 2.66 & $k_{4}$ & 2.71 \\
\hline 29 & $k_{3}$ & 2.54 & $k_{3}$ & 2.50 & $k_{3}$ & 2.48 & $k_{3}$ & 2.44 & $k_{3}$ & 2.46 & $k_{3}$ & 2.41 & $k_{3}$ & 2.45 & $k_{3}$ & 2.44 & $k_{3}$ & 2.28 \\
\hline & $k_{4}$ & 2.43 & $k_{4}$ & 2.48 & $k_{4}$ & 2.51 & $k_{4}$ & 2.55 & $k_{4}$ & 2.52 & $k_{4}$ & 2.57 & $k_{4}$ & 2.53 & $k_{4}$ & 2.54 & $k_{4}$ & 2.75 \\
\hline & $k_{3}$ & 2.53 & $k_{3}$ & 2.50 & $k_{3}$ & 2.47 & $k_{3}$ & 2.47 & $k_{3}$ & 2.37 & $k_{3}$ & 2.43 & $k_{3}$ & 2.42 & $k_{3}$ & 2.42 & $k_{3}$ & 2.17 \\
\hline & $k_{4}$ & 2.44 & $k_{4}$ & 2.47 & $k_{4}$ & 2.51 & $k_{4}$ & 2.51 & $k_{4}$ & 2.63 & $k_{4}$ & 2.55 & $k_{4}$ & 2.56 & $k_{4}$ & 2.56 & $k_{4}$ & 3.02 \\
\hline
\end{tabular}


TABLE 2: The ARL $\mathrm{AR}_{m 1}$ of the EWMA-AM chart $\left(\lambda_{1}=0.05, p_{m 0}=0.1\right.$ with $\mathrm{ARL}_{m 0} \approx 370$ ).

\begin{tabular}{cccccccccc}
\hline$n_{1}$ & & & \multicolumn{7}{c}{$p_{m 1}$} \\
& 0.1 & 0.2 & 0.3 & 0.4 & 0.5 & 0.6 & 0.7 & 0.8 & 0.9 \\
\hline 10 & 370.3 & 10.4 & 4.9 & 3.3 & 2.5 & 2.1 & 1.9 & 1.7 & 1.3 \\
11 & 372.0 & 9.8 & 4.6 & 3.1 & 2.5 & 2.1 & 1.9 & 1.7 & 1.3 \\
12 & 370.4 & 9.3 & 4.4 & 3.0 & 2.3 & 2.0 & 1.8 & 1.4 & 1.1 \\
13 & 370.1 & 8.9 & 4.2 & 2.9 & 2.2 & 1.9 & 1.6 & 1.3 & 1.0 \\
14 & 371.9 & 8.5 & 4.1 & 2.7 & 2.2 & 1.9 & 1.6 & 1.3 & 1.0 \\
15 & 371.3 & 8.2 & 4.0 & 2.7 & 2.1 & 1.8 & 1.5 & 1.2 & 1.0 \\
16 & 370.3 & 7.9 & 3.8 & 2.6 & 2.1 & 1.8 & 1.6 & 1.2 & 1.0 \\
17 & 369.4 & 7.7 & 3.7 & 2.6 & 2.0 & 1.7 & 1.4 & 1.1 & 1.0 \\
18 & 371.0 & 7.4 & 3.7 & 2.5 & 2.0 & 1.6 & 1.3 & 1.1 & 1.0 \\
19 & 368.8 & 7.2 & 3.5 & 2.4 & 2.0 & 1.7 & 1.3 & 1.1 & 1.0 \\
20 & 368.8 & 7.0 & 3.5 & 2.4 & 1.9 & 1.6 & 1.2 & 1.0 & 1.0 \\
21 & 370.2 & 6.8 & 3.4 & 2.4 & 1.9 & 1.7 & 1.3 & 1.0 & 1.0 \\
22 & 369.9 & 6.6 & 3.3 & 2.3 & 1.9 & 1.5 & 1.2 & 1.0 & 1.0 \\
23 & 370.2 & 6.5 & 3.2 & 2.3 & 1.8 & 1.4 & 1.1 & 1.0 & 1.0 \\
24 & 369.9 & 6.3 & 3.1 & 2.2 & 1.9 & 1.5 & 1.2 & 1.0 & 1.0 \\
25 & 370.1 & 6.2 & 3.1 & 2.2 & 1.8 & 1.4 & 1.1 & 1.0 & 1.0 \\
26 & 369.8 & 6.0 & 3.0 & 2.1 & 1.7 & 1.3 & 1.1 & 1.0 & 1.0 \\
27 & 369.8 & 5.9 & 3.0 & 2.2 & 1.8 & 1.4 & 1.1 & 1.0 & 1.0 \\
28 & 369.7 & 5.8 & 2.9 & 2.1 & 1.7 & 1.3 & 1.0 & 1.0 & 1.0 \\
29 & 369.4 & 5.7 & 2.9 & 2.1 & 1.6 & 1.2 & 1.0 & 1.0 & 1.0 \\
30 & 369.9 & 5.6 & 2.9 & 2.1 & 1.7 & 1.3 & 1.0 & 1.0 & 1.0 \\
\hline
\end{tabular}

Similar to the calculation of $\mathrm{ARL}_{m 0}, \mathrm{ARL}_{m 1}$ can be obtained by a Markov chain approach for various $p_{m 1}$ under a specified $p_{m 0}, n_{1}, k_{3}, k_{4}$, and $\lambda_{1}$.

The $\mathrm{ARL}_{m 1}$ s of the EWMA-AM chart under the combinations of $n_{1}=10(1) 30, p_{m 1}=0.1(0.1) 0.9$ with $\mathrm{ARL}_{m 0} \approx 370$ and $\lambda_{1}=0.05$ when $p_{m 0}=0.1,0.3,0.5$ and the corresponding $k_{3}$ and $k_{4}$ are calculated and listed in Tables 2, 3, and 4, respectively.

It can be seen that the values of the $\mathrm{ARL}_{m 1} \mathrm{~s}$ behave appropriately; that is, they change inversely with $n_{1}$, and the $A R L_{m 1}$ s decrease when $p_{m 1}$ is far away from $p_{m 0}$.

When one compares the out-of-control average run lengths between the EWMA-AM chart (Table 4) and the symmetric EWMA mean chart (Table 5) in Yang et al. [17], for example, with $\lambda_{1}=0.05, p_{m 0}=0.5$, and $\mathrm{ARL}_{m 0} \approx 370$, one finds that the EWMA-AM chart shows slightly superior out-of-control detection performance.

\section{The Proposed EWMA-AV Chart}

To monitor the process variance, a random sample of size $n_{2}$, $X_{1}, X_{2}, \ldots, X_{n_{2}}$, is taken from the process, $X$. Assume that the sample size $n_{2}$ is even for convenience (if not, delete one observation).
TABLE 3: The ARL $\mathrm{AR}_{m 1}$ of the EWMA-AM chart $\left(\lambda_{1}=0.05, p_{m 0}=0.3\right.$ with $\mathrm{ARL}_{m 0} \approx 370$ ).

\begin{tabular}{cccccccccc}
\hline$n_{1}$ & & & \multicolumn{7}{c}{$p_{m 1}$} \\
& 0.1 & 0.2 & 0.3 & 0.4 & 0.5 & 0.6 & 0.7 & 0.8 & 0.9 \\
\hline 10 & 7.3 & 17.1 & 373.7 & 17.0 & 7.4 & 4.8 & 3.7 & 2.9 & 2.3 \\
11 & 6.8 & 16.0 & 370.0 & 16.0 & 7.0 & 4.6 & 3.4 & 2.8 & 2.3 \\
12 & 6.5 & 15.1 & 370.1 & 15.1 & 6.7 & 4.4 & 3.4 & 2.8 & 2.2 \\
13 & 6.3 & 14.4 & 369.8 & 14.4 & 6.4 & 4.2 & 3.2 & 2.6 & 2.1 \\
14 & 6.1 & 13.7 & 370.4 & 13.8 & 6.2 & 4.1 & 3.1 & 2.5 & 2.1 \\
15 & 5.9 & 13.1 & 369.9 & 13.2 & 6.0 & 4.0 & 3.0 & 2.4 & 2.0 \\
16 & 5.7 & 12.7 & 369.8 & 12.6 & 5.7 & 3.8 & 2.9 & 2.3 & 2.0 \\
17 & 5.5 & 12.2 & 369.8 & 12.2 & 5.6 & 3.7 & 2.8 & 2.2 & 2.0 \\
18 & 5.4 & 11.7 & 370.1 & 11.8 & 5.4 & 3.6 & 2.7 & 2.2 & 2.0 \\
19 & 5.2 & 11.3 & 370.2 & 11.4 & 5.2 & 3.5 & 2.7 & 2.1 & 2.0 \\
20 & 5.0 & 11.0 & 370.1 & 11.0 & 5.1 & 3.4 & 2.6 & 2.1 & 2.0 \\
21 & 4.9 & 10.7 & 370.1 & 10.7 & 5.0 & 3.3 & 2.5 & 2.1 & 2.0 \\
22 & 4.8 & 10.4 & 370.0 & 10.4 & 4.9 & 3.2 & 2.5 & 2.0 & 2.0 \\
23 & 4.7 & 10.1 & 369.9 & 10.2 & 4.8 & 3.2 & 2.4 & 2.0 & 2.0 \\
24 & 4.6 & 9.9 & 369.7 & 9.9 & 4.7 & 3.1 & 2.4 & 2.0 & 2.0 \\
25 & 4.5 & 9.6 & 370.0 & 9.7 & 4.6 & 3.1 & 2.3 & 2.0 & 2.0 \\
26 & 4.4 & 9.4 & 370.0 & 9.5 & 4.5 & 3.0 & 2.3 & 2.0 & 2.0 \\
27 & 4.3 & 9.2 & 370.5 & 9.3 & 4.4 & 3.0 & 2.2 & 2.0 & 2.0 \\
28 & 4.3 & 9.0 & 370.0 & 9.1 & 4.3 & 2.9 & 2.2 & 2.0 & 1.9 \\
29 & 4.2 & 8.8 & 370.1 & 8.9 & 4.2 & 2.9 & 2.2 & 2.0 & 2.0 \\
30 & 4.1 & 8.7 & 369.8 & 8.7 & 4.2 & 2.8 & 2.2 & 2.0 & 2.0 \\
\hline & & & & & & & & &
\end{tabular}

Define

$$
\begin{aligned}
& Y_{1}^{*}=\frac{\left(X_{2}-X_{1}\right)^{2}}{2}, \\
& Y_{2}^{*}=\frac{\left(X_{4}-X_{3}\right)^{2}}{2}, \\
& Y_{n_{2} / 2}^{*}=\frac{\left(X_{n_{2}}-X_{n_{2}-1}\right)^{2}}{2}, \\
& E\left(Y_{j^{\prime}}^{*}\right)=\sigma^{2}, \quad j^{\prime}=1,2, \ldots, 0.5 n_{2}, \\
& I_{j^{\prime}}=\left\{\begin{array}{ll}
1, & \text { if } Y_{j^{\prime}}^{*}>\sigma^{2} \\
0, & \text { otherwise }
\end{array} \quad \text { for } j^{\prime}=1,2, \ldots, 0.5 n_{2} .\right.
\end{aligned}
$$

Let $V$ be the total number of $Y_{j^{\prime}}^{*}>\sigma^{2}$; then $V=\sum_{j^{\prime}=1}^{0.5 n_{2}} I_{j^{\prime}}$ will have a binomial distribution with parameters $\left(0.5 n_{2}, p_{v 0}\right)$ for an in-control process where $p_{v o}=P\left(Y_{j^{\prime}}^{*}>\sigma^{2}\right)$. The value of $p_{v 0}$ will depend on the distribution of the $X_{i}$ 's. For example, if the $X_{i}$ 's are normally distributed then $p_{v o}=P\left(Y_{j^{\prime}}^{*}>\right.$ $\left.\sigma^{2}\right)=P\left(Z^{2}>1\right)$ where $Z \sim N(0,1)$. Thus in this case $p_{v 0}=0.3147$. If the distribution of $X_{n_{2}}-X_{n_{2}-1}$ is unimodal, as it frequently is, the version of the Tchebychev inequality for 
TABLE 4: The ARL $\mathrm{Al}_{m 1}$ of the EWMA-AM chart $\left(\lambda_{1}=0.05, p_{m 0}=0.5\right.$ with $\mathrm{ARL}_{m 0} \approx 370$ ).

\begin{tabular}{cccccccccc}
\hline$n_{1}$ & & & & & $p_{m 1}$ & & & & \\
& 0.1 & 0.2 & 0.3 & 0.4 & 0.5 & 0.6 & 0.7 & 0.8 & 0.9 \\
\hline 10 & 3.9 & 5.2 & 8.2 & 19.4 & 369.5 & 18.8 & 8.1 & 5.2 & 3.9 \\
11 & 3.7 & 5.0 & 7.7 & 18.2 & 369.9 & 17.7 & 7.6 & 5.0 & 3.7 \\
12 & 3.5 & 4.8 & 7.4 & 17.2 & 369.7 & 16.7 & 7.3 & 4.7 & 3.5 \\
13 & 3.4 & 4.6 & 7.1 & 16.3 & 369.6 & 15.8 & 7.0 & 4.5 & 3.4 \\
14 & 3.2 & 4.4 & 6.8 & 15.5 & 370.3 & 15.2 & 6.7 & 4.4 & 3.2 \\
15 & 3.2 & 4.2 & 6.5 & 15.1 & 370.0 & 14.4 & 6.4 & 4.2 & 3.2 \\
16 & 3.1 & 4.1 & 6.3 & 14.3 & 370.2 & 13.9 & 6.2 & 4.1 & 3.1 \\
17 & 3.1 & 4.0 & 6.1 & 13.7 & 370.0 & 13.4 & 6.0 & 3.9 & 3.0 \\
18 & 3.0 & 3.9 & 5.9 & 13.3 & 370.0 & 12.9 & 5.9 & 3.8 & 3.0 \\
19 & 2.9 & 3.7 & 5.7 & 12.7 & 370.0 & 12.6 & 5.7 & 3.7 & 2.9 \\
20 & 2.9 & 3.7 & 5.6 & 12.4 & 370.0 & 12.1 & 5.5 & 3.7 & 2.9 \\
21 & 2.8 & 3.6 & 5.4 & 12.0 & 369.9 & 11.8 & 5.4 & 3.6 & 2.8 \\
22 & 2.8 & 3.5 & 5.3 & 11.8 & 369.5 & 11.4 & 5.3 & 3.5 & 2.8 \\
23 & 2.7 & 3.4 & 5.2 & 11.4 & 369.9 & 11.2 & 5.2 & 3.4 & 2.7 \\
24 & 2.7 & 3.4 & 5.1 & 11.1 & 370.0 & 10.9 & 5.0 & 3.4 & 2.7 \\
25 & 2.6 & 3.3 & 5.0 & 10.8 & 370.0 & 10.7 & 4.9 & 3.3 & 2.6 \\
26 & 2.4 & 3.3 & 4.9 & 10.5 & 370.3 & 10.4 & 4.8 & 3.3 & 2.4 \\
27 & 2.5 & 3.2 & 4.8 & 10.2 & 369.3 & 10.2 & 4.8 & 3.2 & 2.5 \\
28 & 2.3 & 3.2 & 4.8 & 10.4 & 369.9 & 9.8 & 4.7 & 3.2 & 2.3 \\
29 & 2.3 & 3.1 & 4.6 & 9.9 & 370.0 & 9.7 & 4.6 & 3.1 & 2.2 \\
30 & 2.2 & 3.1 & 4.6 & 9.9 & 369.8 & 9.5 & 4.5 & 3.1 & 2.2 \\
\hline
\end{tabular}

unimodal variables implies that the quantity $p_{v 0}$ is bounded above by $4 / 9$. The value of $p_{v 0}$ can be arbitrarily small but it usually will be in the range $0.25-0.50$. Note that, although the resulting chart is a $0.5 n_{2} p_{v 0}$ chart, this is a new chart in that the binomial variable is not the count of nonconforming units in the sample but rather the number of pairs of $X$ values in a sample that is in control with respect to the process variance. Monitoring process variance shifts are equivalent to monitoring the changes in process proportion, $p_{v 0}$. For the incontrol process, we may define the monitoring statistic $V_{t}$ as the number of $Y_{j}^{*}$ 's $>\sigma^{2}$ at time $t$; hence, $V_{t} \sim B\left(0.5 n_{2}, p_{v 0}\right)$.

3.1. The Control Limits of EWMA-AV Chart. The variance chart based on an asymmetric binomial distribution has similar defects to those of the corresponding mean chart. It exhibits an irregular in-control average run length and its out-of-control average run length does not change appropriately as sample size increases. A new EWMA asymmetric variance chart (EWMA-AV chart) based on the statistic $V$ is constructed as follows:

$$
\begin{aligned}
& \mathrm{UCL}_{\mathrm{EWMA}_{V}}= 0.5 n_{2} p_{v 0}+L_{3} \sqrt{\frac{\lambda_{2}}{\left(2-\lambda_{2}\right)} 0.5 n_{2} p_{v 0}\left(1-p_{v 0}\right)} \\
& \mathrm{CL}_{\mathrm{EWMA}_{V}}=0.5 n_{2} p_{0 v}, \\
& \mathrm{LCL}_{\mathrm{EWMA}_{V}}=0.5 n_{2} p_{v 0}-L_{4} \sqrt{\frac{\lambda_{2}}{\left(2-\lambda_{2}\right)} 0.5 n_{2} p_{v 0}\left(1-p_{v 0}\right)}
\end{aligned}
$$

TABLE 5: The ARL $\mathrm{Af}_{1}$ of the symmetric EWMA mean chart $\left(\lambda_{1}=0.05\right.$, $p_{m 0}=0.5$ with $\mathrm{ARL}_{m 0} \approx 370$ ).

\begin{tabular}{cccccccccc}
\hline$n_{1}$ & & & & \multicolumn{7}{c}{$p_{m 1}$} & & & & \\
& 0.1 & 0.2 & 0.3 & 0.4 & 0.5 & 0.6 & 0.7 & 0.8 & 0.9 \\
\hline 10 & 4 & 5 & 8 & 19 & 371 & 19 & 8 & 5 & 4 \\
11 & 4 & 5 & 8 & 18 & 370 & 18 & 8 & 5 & 4 \\
12 & 4 & 5 & 7 & 17 & 380 & 17 & 7 & 5 & 4 \\
13 & 3 & 5 & 7 & 16 & 377 & 16 & 7 & 5 & 3 \\
14 & 3 & 4 & 7 & 15 & 378 & 15 & 7 & 4 & 3 \\
15 & 3 & 4 & 7 & 15 & 386 & 15 & 7 & 4 & 3 \\
16 & 3 & 4 & 6 & 14 & 371 & 14 & 6 & 4 & 3 \\
17 & 3 & 4 & 6 & 14 & 384 & 14 & 6 & 4 & 3 \\
18 & 3 & 4 & 6 & 13 & 375 & 13 & 6 & 4 & 3 \\
19 & 3 & 4 & 6 & 13 & 388 & 13 & 6 & 4 & 3 \\
20 & 3 & 4 & 6 & 12 & 389 & 12 & 6 & 4 & 3 \\
21 & 3 & 4 & 5 & 12 & 379 & 12 & 5 & 4 & 3 \\
22 & 3 & 4 & 5 & 12 & 383 & 12 & 5 & 4 & 3 \\
23 & 3 & 4 & 5 & 11 & 383 & 11 & 5 & 3 & 3 \\
24 & 3 & 3 & 5 & 11 & 381 & 11 & 5 & 3 & 3 \\
25 & 3 & 3 & 5 & 11 & 377 & 11 & 5 & 3 & 3 \\
\hline
\end{tabular}

where $L_{3}$ and $L_{4}$ are appropriately chosen coefficients for $\mathrm{UCL}_{\mathrm{EWMA}_{V}}$ and $\mathrm{LCL}_{\mathrm{EWMA}_{V}}$.

If any monitoring statistic $\mathrm{EWMA}_{V_{t}}$ exceeds $\mathrm{UCL}_{\mathrm{EWMA}_{V}}$ or if $\mathrm{EWMA}_{V_{t}}<\mathrm{LCL}_{\mathrm{EWMA}_{V}}$, the process is deemed to show some out-of-variance-control signals.

The monitoring statistic EWMA $V_{t}$ based on the statistic $V_{t}$ is thus defined as follows:

$$
\begin{array}{r}
\mathrm{EWMA}_{V_{t}}=\lambda_{2} V_{t}+\left(1-\lambda_{2}\right) \mathrm{EWMA}_{V_{t-1}} \\
0<\lambda_{2} \leq 1, \quad t=1,2, \ldots .
\end{array}
$$

Let the starting value, $\mathrm{EWMA}_{V_{0}}$, be the mean of $V$; that is $\mathrm{EWMA}_{V_{0}}=n_{2} p_{\nu 0}$ for an in-control process. Hence the mean and variance of $\mathrm{EWMA}_{V_{t}}$ are

$$
\begin{gathered}
E\left(\operatorname{EWMA}_{V_{t}}\right)=0.5 n_{2} p_{v 0} \\
\operatorname{Var}\left(\mathrm{EWMA}_{V_{t}}\right)=\frac{\lambda_{2}\left[1-\left(1-\lambda_{2}\right)^{2 t}\right]}{2-\lambda_{2}}\left(0.5 n_{2} p_{v 0}\left(1-p_{v 0}\right)\right) .
\end{gathered}
$$

The asymptotic variance of $\mathrm{EWMA}_{V_{t}}$ is

$$
\operatorname{Var}\left(\mathrm{EWMA}_{V_{t}}\right)=\frac{\lambda_{2}}{2-\lambda_{2}}\left(0.5 n_{2} p_{v 0}\left(1-p_{v 0}\right)\right)
$$

The ARL is also used to measure the performance of the proposed new EWMA-AV chart. The in-control ARL, ARL $L_{v 0}$, of the EWMA-AV chart depends on the values of $n_{2}, L_{3}, L_{4}$, $\lambda_{2}$, and $p_{v 0}$. In a manner similar to that used to obtain $k_{3}$ and $k_{4}$ in Section 2, the two parameters, $L_{3}$ and $L_{4}$, may be obtained when the values of $0.5 n_{2}$ and $p_{v 0}$ are given and hence 
TABLE 6: The $L_{3}$ and $L_{4}$ with $\mathrm{ARL}_{v 0} \approx 370$ for various $0.5 n_{2}$ and $p_{v 0}$ given $\lambda_{2}=0.05$.

\begin{tabular}{|c|c|c|c|c|c|c|c|c|}
\hline \multirow{4}{*}{$\begin{array}{l}0.5 n_{2} \\
5\end{array}$} & \multicolumn{8}{|c|}{$p_{v 0}$} \\
\hline & \multicolumn{2}{|c|}{0.10} & \multicolumn{2}{|c|}{0.20} & \multicolumn{2}{|c|}{0.30} & \multicolumn{2}{|c|}{0.40} \\
\hline & $L_{3}$ & 2.69 & $L_{3}$ & 2.58 & $L_{3}$ & 2.51 & $L_{3}$ & 2.49 \\
\hline & $L_{4}$ & 2.26 & $L_{4}$ & 2.39 & $L_{4}$ & 2.45 & $L_{4}$ & 2.48 \\
\hline \multirow{2}{*}{6} & $L_{3}$ & 2.66 & $L_{3}$ & 2.58 & $L_{3}$ & 2.51 & $L_{3}$ & 2.48 \\
\hline & $L_{4}$ & 2.28 & $L_{4}$ & 2.39 & $L_{4}$ & 2.46 & $L_{4}$ & 2.48 \\
\hline \multirow{2}{*}{7} & $L_{3}$ & 2.62 & $L_{3}$ & 2.55 & $L_{3}$ & 2.52 & $L_{3}$ & 2.47 \\
\hline & $L_{4}$ & 2.31 & $L_{4}$ & 2.39 & $L_{4}$ & 2.46 & $L_{4}$ & 2.52 \\
\hline \multirow{2}{*}{8} & $L_{3}$ & 2.63 & $L_{3}$ & 2.54 & $L_{3}$ & 2.49 & $L_{3}$ & 2.47 \\
\hline & $L_{4}$ & 2.32 & $L_{4}$ & 2.42 & $L_{4}$ & 2.48 & $L_{4}$ & 2.51 \\
\hline \multirow{2}{*}{9} & $L_{3}$ & 2.62 & $L_{3}$ & 2.54 & $L_{3}$ & 2.52 & $L_{3}$ & 2.45 \\
\hline & $L_{4}$ & 2.34 & $L_{4}$ & 2.43 & $L_{4}$ & 2.45 & $L_{4}$ & 2.53 \\
\hline \multirow{2}{*}{10} & $L_{3}$ & 2.61 & $L_{3}$ & 2.54 & $L_{3}$ & 2.49 & $L_{3}$ & 2.46 \\
\hline & $L_{4}$ & 2.36 & $L_{4}$ & 2.44 & $L_{4}$ & 2.49 & $L_{4}$ & 2.53 \\
\hline \multirow{2}{*}{11} & $L_{3}$ & 2.61 & $L_{3}$ & 2.54 & $L_{3}$ & 2.49 & $L_{3}$ & 2.44 \\
\hline & $L_{4}$ & 2.36 & $L_{4}$ & 2.44 & $L_{4}$ & 2.48 & $L_{4}$ & 2.54 \\
\hline \multirow{2}{*}{12} & $L_{3}$ & 2.60 & $L_{3}$ & 2.53 & $L_{3}$ & 2.49 & $L_{3}$ & 2.45 \\
\hline & $L_{4}$ & 2.36 & $L_{4}$ & 2.44 & $L_{4}$ & 2.48 & $L_{4}$ & 2.53 \\
\hline \multirow{2}{*}{13} & $L_{3}$ & 2.58 & $L_{3}$ & 2.53 & $L_{3}$ & 2.48 & $L_{3}$ & 2.45 \\
\hline & $L_{4}$ & 2.38 & $L_{4}$ & 2.43 & $L_{4}$ & 2.50 & $L_{4}$ & 2.53 \\
\hline \multirow{2}{*}{14} & $L_{3}$ & 2.58 & $L_{3}$ & 2.51 & $L_{3}$ & 2.49 & $L_{3}$ & 2.47 \\
\hline & $L_{4}$ & 2.35 & $L_{4}$ & 2.47 & $L_{4}$ & 2.49 & $L_{4}$ & 2.50 \\
\hline \multirow{2}{*}{15} & $L_{3}$ & 2.57 & $L_{3}$ & 2.51 & $L_{3}$ & 2.49 & $L_{3}$ & 2.47 \\
\hline & $L_{4}$ & 2.37 & $L_{4}$ & 2.47 & $L_{4}$ & 2.49 & $L_{4}$ & 2.51 \\
\hline
\end{tabular}

one sets $\mathrm{ARL}_{v 0} \approx 370$ using Markov chain approach. The $L_{3}$ and $L_{4}$ with $\lambda_{2}=0.05$ and $\mathrm{ARL}_{v 0} \approx 370$ for $0.5 n_{2}=5(1) 15$ and $p_{v 0}=0.1(0.1) 0.4$ are illustrated in Table 6 .

3.2. The Out-of-Control Average Run Lengths of the EWMA$A V$ Chart. To measure the out-of-control detection performance of the EWMA-AV chart, we calculate the out-ofcontrol average run length $\left(\mathrm{ARL}_{v 1}\right)$ for the combinations of $0.5 n_{2}=5(1) 15$, out-of-control proportion $p_{v 1}=0.1(0.1) 0.9$ with $\mathrm{ARL}_{v 0} \approx 370$ and $\lambda_{2}=0.05$ for adopting $p_{v 0}=0.1,0.4$, and corresponding $L_{3}$ and $L_{4}$ using Markov chain approach. The results are listed in Tables 7 and 8.

The results look reasonable since the values of the $\mathrm{ARL}_{v 1} \mathrm{~S}$ change inversely with $n_{2}$, and the $\mathrm{ARL}_{v 1}$ s decrease when $p_{v 1}$ is far away from $p_{v 0}$.

\section{Performance Measurement of Using EWMA-AM Chart and EWMA-AV Chart Simultaneously}

Using both the EWMA-AM chart and EWMA-AV chart we may monitor the process mean and variance simultaneously. We will use the overall average run length (ARL) to measure the performance of using both the EWMA-AM chart and EWMA-AV chart.
TABLE 7: The ARL $\mathrm{Al}_{v 1}$ of the EWMA-AV chart for $\lambda_{2}=0.05$ and $p_{v 0}=$ 0.1 .

\begin{tabular}{lccccccccc}
\hline \multirow{2}{*}{$0.5 n_{2}$} & & \multicolumn{8}{c}{$p_{v 1}$} \\
& 0.1 & 0.2 & 0.3 & 0.4 & 0.5 & 0.6 & 0.7 & 0.8 & 0.9 \\
\hline 5 & 375.3 & 16.1 & 7.1 & 4.7 & 3.5 & 2.9 & 2.4 & 2.1 & 2.0 \\
6 & 374.2 & 14.3 & 6.4 & 4.2 & 3.2 & 2.7 & 2.3 & 2.1 & 2.0 \\
7 & 370.1 & 13.0 & 5.9 & 3.8 & 2.8 & 2.3 & 2.0 & 1.9 & 1.6 \\
8 & 369.9 & 12.0 & 5.5 & 3.6 & 2.8 & 2.3 & 2.0 & 1.9 & 1.6 \\
9 & 368.8 & 11.1 & 5.2 & 3.5 & 2.7 & 2.3 & 2.0 & 1.9 & 1.6 \\
10 & 370.3 & 10.4 & 4.9 & 3.3 & 2.5 & 2.1 & 1.9 & 1.7 & 1.3 \\
11 & 372.0 & 9.8 & 4.6 & 3.1 & 2.5 & 2.1 & 1.9 & 1.7 & 1.3 \\
12 & 370.4 & 9.3 & 4.4 & 3.0 & 2.3 & 2.0 & 1.8 & 1.4 & 1.1 \\
13 & 370.1 & 8.9 & 4.2 & 2.9 & 2.2 & 1.9 & 1.6 & 1.3 & 1.0 \\
14 & 371.9 & 8.5 & 4.1 & 2.7 & 2.2 & 1.9 & 1.6 & 1.3 & 1.0 \\
15 & 371.3 & 8.2 & 4.0 & 2.7 & 2.1 & 1.8 & 1.5 & 1.2 & 1.0 \\
\hline
\end{tabular}

TABLE 8: The ARL $\mathrm{Al}_{v 1}$ of the EWMA-AV chart for $\lambda_{2}=0.05$ and $p_{v 0}=$ 0.4 .

\begin{tabular}{lccccccccc}
\hline $0.5 n_{2}$ & & & \multicolumn{7}{c}{$p_{v 1}$} \\
& 0.1 & 0.2 & 0.3 & 0.4 & 0.5 & 0.6 & 0.7 & 0.8 & 0.9 \\
\hline 5 & 7.2 & 11.8 & 30.2 & 370.1 & 29.8 & 11.9 & 7.4 & 5.4 & 4.4 \\
6 & 6.6 & 10.6 & 26.6 & 370.5 & 26.2 & 10.7 & 6.7 & 4.9 & 4.0 \\
7 & 6.1 & 9.7 & 24.0 & 370.0 & 23.3 & 9.7 & 6.2 & 4.6 & 3.7 \\
8 & 5.8 & 9.0 & 21.8 & 369.6 & 21.4 & 9.0 & 5.7 & 4.3 & 3.4 \\
9 & 5.4 & 8.4 & 20.4 & 370.1 & 19.6 & 8.4 & 5.4 & 4.0 & 3.3 \\
10 & 5.1 & 7.9 & 18.9 & 369.9 & 18.4 & 7.9 & 5.1 & 3.8 & 3.1 \\
11 & 4.9 & 7.5 & 17.7 & 369.9 & 17.2 & 7.5 & 4.9 & 3.6 & 3.0 \\
12 & 4.6 & 7.2 & 16.7 & 370.0 & 16.3 & 7.1 & 4.6 & 3.5 & 2.9 \\
13 & 4.5 & 6.8 & 15.8 & 369.7 & 15.5 & 6.9 & 4.5 & 3.4 & 2.8 \\
14 & 4.3 & 6.6 & 15.0 & 370.1 & 14.9 & 6.6 & 4.3 & 3.3 & 2.8 \\
15 & 4.1 & 6.3 & 14.3 & 370.1 & 14.3 & 6.4 & 4.2 & 3.2 & 2.6 \\
\hline
\end{tabular}

Since the statistics $\mathrm{EWMA}_{M}$ and $\mathrm{EWMA}_{V}$ are independent, the in-control overall $A R L, A R L_{0}$, of the newly proposed EWMA-AM and EWMA-AV charts is well approximated as follows (see Hawkins (1992)):

$$
\begin{aligned}
& \mathrm{ARL}_{0} \\
& =\frac{1}{1 / \mathrm{ARL}_{m 0}+1 / \mathrm{ARL}_{v 0}-\left(\left(1 / \mathrm{ARL}_{m 0}\right)\left(1 / \mathrm{ARL}_{v 0}\right)\right)} \\
& \approx \frac{1}{1 / \mathrm{ARL}_{m 0}+1 / \mathrm{ARL}_{v 0}} .
\end{aligned}
$$

The $\mathrm{ARL}_{0}$ of using both the EWMA-AM chart and EWMA-AV chart with any combinations of $\left(n_{1}, n_{2}\right)$ and $\left(p_{m 0}, p_{v 0}\right)$ are all approximately 185 because of

$$
\mathrm{ARL}_{0} \approx \frac{1}{1 / \mathrm{ARL}_{m 0}+1 / \mathrm{ARL}_{v 0}}=\frac{1}{1 / 370+1 / 370}
$$

If the process is out-of-control because the mean $\mu$ has shifted, the process proportion becomes $p_{m 1}\left(\neq p_{m 0}\right)$. If 
TABLE 9: The ARL $\mathrm{AR}_{1}$ of the EWMA-AM and EWMA-AV charts for $\lambda_{1}=\lambda_{2}=0.05$ and $p_{m 0}=p_{v 0}=0.1$.

\begin{tabular}{cccccccccccc}
\hline & \multicolumn{4}{c}{$p_{v 1}=0.1$} & \multicolumn{3}{c}{$p_{v 1}=0.3$} & \multicolumn{3}{c}{$p_{v 1}=0.7$} \\
$n_{1}$ & $0.5 n_{2}$ & \multicolumn{3}{c}{$p_{m 1}$} & \multicolumn{3}{c}{$p_{m 1}$} & & & $p_{m 1}$ \\
& & 0.1 & 0.3 & 0.7 & 0.1 & 0.3 & 0.7 & 0.1 & 0.3 & 0.7 \\
\hline 10 & 5 & 186.4 & 4.8 & 1.9 & 7.0 & 2.9 & 1.5 & 2.4 & 1.6 & 1.1 \\
12 & 6 & 186.1 & 4.3 & 1.8 & 6.3 & 2.6 & 1.4 & 2.3 & 1.5 & 1.0 \\
14 & 7 & 185.5 & 4.1 & 1.6 & 5.8 & 2.4 & 1.2 & 2.0 & 1.3 & 0.9 \\
16 & 8 & 185.0 & 3.8 & 1.6 & 5.4 & 2.2 & 1.2 & 2.0 & 1.3 & 0.9 \\
18 & 9 & 185.0 & 3.7 & 1.3 & 5.1 & 2.2 & 1.0 & 2.0 & 1.3 & 0.8 \\
20 & 10 & 184.8 & 3.5 & 1.2 & 4.8 & 2.0 & 1.0 & 1.9 & 1.2 & 0.7 \\
22 & 11 & 185.5 & 3.3 & 1.2 & 4.5 & 1.9 & 1.0 & 1.9 & 1.2 & 0.7 \\
24 & 12 & 185.1 & 3.1 & 1.2 & 4.3 & 1.8 & 0.9 & 1.8 & 1.1 & 0.7 \\
26 & 13 & 185.0 & 3.0 & 1.1 & 4.2 & 1.8 & 0.9 & 1.6 & 1.0 & 0.7 \\
28 & 14 & 185.4 & 2.9 & 1.0 & 4.1 & 1.7 & 0.8 & 1.6 & 1.0 & 0.6 \\
30 & 15 & 185.3 & 2.9 & 1.0 & 4.0 & 1.7 & 0.8 & 1.5 & 1.0 & 0.6 \\
\hline
\end{tabular}

TABLE 10: The ARL $\mathrm{A}_{1}$ of the EWMA-AM and EWMA-AV charts for $\lambda_{1}=\lambda_{2}=0.05, p_{m 0}=0.3$, and $p_{v 0}=0.1$.

\begin{tabular}{cccccccccccc}
\hline & \multicolumn{4}{c}{$p_{v 1}=0.1$} & \multicolumn{3}{c}{$p_{v 1}=0.3$} & \multicolumn{3}{c}{$p_{v 1}=0.7$} \\
$n_{1}$ & $0.5 n_{2}$ & \multicolumn{3}{c}{$p_{m 1}$} & \multicolumn{3}{c}{$p_{m 1}$} & & & $p_{m 1}$ \\
& & 0.1 & 0.3 & 0.7 & 0.1 & 0.3 & 0.7 & 0.1 & 0.3 & 0.7 \\
\hline 10 & 5 & 7.2 & 187.2 & 3.7 & 3.6 & 7.0 & 2.4 & 1.8 & 2.4 & 1.5 \\
12 & 6 & 6.4 & 186.1 & 3.4 & 3.2 & 6.3 & 2.2 & 1.7 & 2.3 & 1.4 \\
14 & 7 & 6.0 & 185.1 & 3.1 & 3.0 & 5.8 & 2.0 & 1.5 & 2.0 & 1.2 \\
16 & 8 & 5.6 & 185.0 & 2.9 & 2.8 & 5.4 & 1.9 & 1.5 & 2.0 & 1.2 \\
18 & 9 & 5.3 & 184.7 & 2.7 & 2.7 & 5.1 & 1.8 & 1.5 & 2.0 & 1.1 \\
20 & 10 & 4.9 & 185.1 & 2.6 & 2.5 & 4.8 & 1.7 & 1.4 & 1.9 & 1.1 \\
22 & 11 & 4.7 & 185.5 & 2.5 & 2.3 & 4.5 & 1.6 & 1.4 & 1.9 & 1.1 \\
24 & 12 & 4.5 & 185.0 & 2.4 & 2.2 & 4.3 & 1.6 & 1.3 & 1.8 & 1.0 \\
26 & 13 & 4.3 & 185.0 & 2.3 & 2.1 & 4.2 & 1.5 & 1.2 & 1.6 & 0.9 \\
28 & 14 & 4.3 & 185.5 & 2.2 & 2.1 & 4.1 & 1.4 & 1.2 & 1.6 & 0.9 \\
30 & 15 & 4.1 & 185.3 & 2.2 & 2.0 & 4.0 & 1.4 & 1.1 & 1.5 & 0.9 \\
\hline
\end{tabular}

the process is out-of-control because the variance $\sigma^{2}$ has changed, the process proportion becomes $p_{v 1}\left(\neq p_{v 0}\right)$.

The out-of-control ARL, $\mathrm{ARL}_{1}$, when using the EWMAAM chart and EWMA-AV chart simultaneously can be calculated approximately using

$$
\mathrm{ARL}_{1} \approx \frac{1}{1 / \mathrm{ARL}_{m 1}+1 / \mathrm{ARL}_{v 1}} .
$$

We calculate approximate $\mathrm{ARL}_{1} \mathrm{~s}$ for the combinations of $n_{1}=10(2) 30,0.5 n_{2}=5(1) 15, p_{m 1}=0.1,0.3,0.7$, and $p_{v 1}=$ $0.1,0.3,0.7$ with $p_{m 0}=0.1, p_{v 0}=0.1, \lambda_{1}=\lambda_{2}=0.05$, and $\mathrm{ARL}_{0} \approx 185$. The results are listed in Table 9. The $\mathrm{ARL}_{1} \mathrm{~s}$ for the combinations of $n_{1}=10(2) 30,0.5 n_{2}=5(1) 15, p_{m 1}=$ $0.1,0.3,0.7$, and $p_{v 1}=0.1,0.3,0.7$ with $p_{m 0}=0.3, p_{v 0}=0.1$, $\mathrm{ARL}_{0} \approx 185$, and $\lambda_{1}=\lambda_{2}=0.05$ are calculated and listed in
TABLE 11: The ARL ${ }_{1}$ of the EWMA-AM and EWMA-AV charts for $\lambda_{1}=\lambda_{2}=0.05, p_{m 0}=0.5$, and $p_{v 0}=0.4$.

\begin{tabular}{cccccccccccc}
\hline & \multicolumn{4}{c}{$p_{v 1}=0.1$} & \multicolumn{3}{c}{$p_{v 1}=0.3$} & \multicolumn{3}{c}{$p_{v 1}=0.7$} \\
$n_{1}$ & $0.5 n_{2}$ & \multicolumn{3}{c}{$p_{m 1}$} & \multicolumn{3}{c}{$p_{m 1}$} & & & $p_{m 1}$ \\
& & 0.1 & 0.3 & 0.7 & 0.1 & 0.3 & 0.7 & 0.1 & 0.3 & 0.7 \\
\hline 10 & 5 & 2.6 & 3.8 & 3.8 & 3.4 & 6.4 & 6.4 & 2.6 & 3.9 & 3.9 \\
12 & 6 & 2.3 & 3.5 & 3.5 & 3.1 & 5.8 & 5.7 & 2.3 & 3.5 & 3.5 \\
14 & 7 & 2.1 & 3.2 & 3.2 & 2.8 & 5.3 & 5.2 & 2.1 & 3.2 & 3.2 \\
16 & 8 & 2.0 & 3.0 & 3.0 & 2.7 & 4.9 & 4.8 & 2.0 & 3.0 & 3.0 \\
18 & 9 & 1.9 & 2.8 & 2.8 & 2.6 & 4.6 & 4.6 & 1.9 & 2.8 & 2.8 \\
20 & 10 & 1.8 & 2.7 & 2.6 & 2.5 & 4.3 & 4.2 & 1.8 & 2.7 & 2.6 \\
22 & 11 & 1.8 & 2.5 & 2.5 & 2.4 & 4.1 & 4.1 & 1.8 & 2.5 & 2.5 \\
24 & 12 & 1.7 & 2.4 & 2.4 & 2.3 & 3.9 & 3.8 & 1.7 & 2.4 & 2.4 \\
26 & 13 & 1.6 & 2.3 & 2.3 & 2.1 & 3.7 & 3.7 & 1.6 & 2.3 & 2.3 \\
28 & 14 & 1.5 & 2.3 & 2.2 & 2.0 & 3.6 & 3.6 & 1.5 & 2.3 & 2.2 \\
30 & 15 & 1.4 & 2.2 & 2.1 & 1.9 & 3.5 & 3.4 & 1.4 & 2.2 & 2.2 \\
\hline
\end{tabular}

Table 10. The $\mathrm{ARL}_{1} \mathrm{~s}$ for the combinations of $n_{1}=10(2) 30$, $0.5 n_{2}=5(1) 15, p_{m 1}=0.1,0.3,0.7$, and $p_{v 1}=0.1,0.3,0.7$ with $p_{m 0}=0.5, p_{\nu 0}=0.4, \mathrm{ARL}_{0} \approx 185$, and $\lambda_{1}=\lambda_{2}=$ 0.05 are calculated and listed in Table 11. In Tables 9-11, we observe that the $\mathrm{ARL}_{1}$ changes inversely with $n_{1}$ and $n_{2}$, and the $\mathrm{ARL}_{1}$ decreases when $p_{m 1}$ is far away from $p_{m 0}$ and/or $p_{v 1}$ is far away from $p_{v 0}$. This contrasts with the previously noted inappropriate behavior exhibited by the $V$ and the $M$ charts.

\section{When Population Mean and Variance Are Unknown}

When the in-control process mean, $\mu$, and the process variance, $\sigma^{2}$, are unknown, and hence the in-control process proportions, $p_{m 0}$ and $p_{v 0}$, are unknown, we can use the following two preliminary independent sample data sets:

$$
\begin{gathered}
X_{t 1}, X_{t 2}, \ldots, X_{t n_{1}}, \quad t=1,2, \ldots, k, \\
X_{t n_{1}+1}, X_{t n_{1}+2}, \ldots, X_{t n_{1}+n_{2}}, \quad t=1,2, \ldots, k
\end{gathered}
$$

from $k$ sampling periods, each with an even number of observations, $n_{1}$ and $n_{2}$, to estimate them (see, e.g., [20]); that is

$$
\widehat{\mu}=\overline{\bar{x}}=\frac{\sum_{t=1}^{k} \sum_{j=1}^{n_{1}} x_{t j}}{k n_{1}}, \quad \widehat{\sigma}=\frac{\bar{S}}{c_{4}}=\frac{\sum_{t=1}^{k} S_{t}}{c_{4} k},
$$


TABLE 12: (a) The service times from the first ten counters in a bank branch $\left(\lambda_{1}=\lambda_{2}=0.05\right)$. (b) The service times from the last ten counters in a bank branch $\left(\lambda_{1}=\lambda_{2}=0.05\right)$.

(a)

\begin{tabular}{cccccccccccccc}
\hline$t$ & $X_{1}$ & $X_{2}$ & $X_{3}$ & $X_{4}$ & $X_{5}$ & $X_{6}$ & $X_{7}$ & $X_{8}$ & $X_{9}$ & $X_{10}$ & $M_{t}$ & EWMA $_{M_{i}}$ \\
\hline 1 & 0.88 & 0.78 & 5.06 & 5.45 & 2.93 & 6.11 & 11.59 & 1.20 & 0.89 & 3.21 & 2 & 3.81 \\
2 & 3.82 & 13.4 & 5.16 & 3.20 & 32.27 & 3.68 & 3.14 & 1.58 & 2.72 & 7.71 & 3 & 3.76 \\
3 & 1.40 & 3.89 & 10.88 & 30.85 & 0.54 & 8.40 & 5.10 & 2.63 & 9.17 & 3.94 & 4 & 3.78 \\
4 & 16.8 & 8.77 & 8.36 & 3.55 & 7.76 & 1.81 & 1.11 & 5.91 & 8.26 & 7.19 & 7 & 3.94 \\
5 & 0.24 & 9.57 & 0.66 & 1.15 & 2.34 & 0.57 & 8.94 & 5.54 & 11.69 & 6.58 & 4 & 3.94 \\
6 & 4.21 & 8.73 & 11.44 & 2.89 & 19.49 & 1.20 & 8.01 & 6.19 & 7.48 & 0.07 & 6 & 4.04 \\
7 & 15.08 & 7.43 & 4.31 & 6.14 & 10.37 & 2.33 & 1.97 & 1.08 & 4.27 & 14.08 & 5 & 4.09 \\
8 & 13.89 & 0.30 & 3.21 & 11.32 & 9.90 & 4.39 & 10.5 & 1.70 & 10.74 & 1.46 & 5 & 4.14 \\
9 & 0.03 & 12.76 & 2.41 & 7.41 & 1.67 & 3.70 & 4.31 & 2.45 & 3.57 & 3.33 & 2 & 4.03 \\
10 & 12.89 & 17.96 & 2.78 & 3.21 & 1.12 & 12.61 & 4.23 & 6.18 & 2.33 & 6.92 & 5 & 4.08 \\
11 & 7.71 & 1.05 & 1.11 & 0.22 & 3.53 & 0.81 & 0.41 & 3.73 & 0.08 & 2.55 & 1 & 3.92 \\
12 & 5.81 & 6.29 & 3.46 & 2.66 & 4.02 & 10.95 & 1.59 & 5.58 & 0.55 & 4.10 & 3 & 3.88 \\
13 & 2.89 & 1.61 & 1.30 & 2.58 & 18.65 & 10.77 & 18.23 & 3.13 & 3.38 & 6.34 & 4 & 3.88 \\
14 & 1.36 & 1.92 & 0.12 & 11.08 & 8.85 & 3.99 & 4.32 & 1.71 & 1.77 & 1.94 & 2 & 3.79 \\
15 & 21.52 & 0.63 & 8.54 & 3.37 & 6.94 & 3.44 & 3.37 & 6.37 & 1.28 & 12.83 & 5 & 3.85 \\
\hline
\end{tabular}

(b)

\begin{tabular}{cccccccccccccc}
\hline$t$ & $X_{11}$ & $X_{12}$ & $X_{13}$ & $X_{14}$ & $X_{15}$ & $X_{16}$ & $X_{17}$ & $X_{18}$ & $X_{19}$ & $X_{20}$ & $V_{t}$ & $\mathrm{EWMA}_{V_{i}}$ \\
\hline 1 & 3.82 & 6.29 & 10.88 & 30.85 & 9.9 & 3.99 & 1.59 & 1.71 & 8.26 & 4.1 & 1 \\
2 & 0.24 & 12.76 & 11.44 & 3.2 & 3.53 & 0.57 & 18.23 & 2.45 & 2.72 & 6.92 & 3 & 1.19 \\
3 & 3.82 & 7.43 & 0.12 & 3.37 & 1.12 & 12.61 & 1.59 & 1.08 & 0.89 & 0.07 & 1 & 1.27 \\
4 & 13.89 & 3.89 & 5.16 & 11.32 & 4.02 & 0.57 & 8.01 & 6.19 & 1.77 & 6.58 & 1 & 1.25 \\
5 & 5.81 & 12.76 & 2.41 & 1.15 & 3.53 & 0.81 & 11.59 & 5.91 & 4.27 & 3.33 & 0 & 1.19 \\
6 & 12.89 & 8.73 & 10.88 & 2.89 & 18.65 & 10.95 & 0.41 & 3.13 & 4.27 & 7.71 & 1 & 1.18 \\
7 & 2.89 & 0.63 & 0.12 & 0.22 & 4.02 & 10.95 & 8.01 & 1.08 & 10.74 & 4.1 & 0 \\
8 & 16.8 & 1.05 & 1.3 & 3.2 & 2.34 & 0.81 & 4.32 & 3.13 & 0.08 & 1.46 & 1 & 1.12 \\
9 & 4.21 & 17.96 & 5.06 & 0.22 & 4.02 & 3.99 & 8.01 & 5.91 & 0.55 & 3.33 & 1 & 1.12 \\
10 & 12.89 & 8.77 & 11.44 & 7.41 & 1.12 & 1.81 & 4.32 & 5.58 & 0.89 & 14.08 & 1 & 1.10 \\
11 & 0.88 & 8.77 & 5.06 & 3.55 & 8.85 & 10.95 & 18.23 & 5.54 & 2.33 & 6.58 & 2 \\
12 & 7.71 & 7.43 & 0.12 & 2.58 & 1.12 & 2.33 & 4.23 & 2.63 & 4.27 & 3.33 & 0 & 1.15 \\
13 & 7.71 & 9.57 & 0.12 & 30.85 & 7.76 & 1.81 & 3.14 & 1.71 & 2.72 & 14.08 & 2 \\
14 & 2.89 & 1.05 & 2.41 & 11.32 & 32.27 & 8.4 & 1.97 & 2.45 & 11.69 & 12.83 & 2 \\
15 & 1.36 & 0.63 & 3.46 & 11.32 & 0.54 & 10.95 & 4.23 & 2.45 & 2.33 & 6.34 & 2 & 1.14 & 1.22 \\
\hline
\end{tabular}

where

$$
\begin{gathered}
\widehat{p}_{m 0}=\frac{\sum_{t=1}^{k}\left(M_{t} / n_{1}\right)}{k}, \quad \widehat{p}_{v 0}=\frac{\sum_{t=1}^{k}\left(V_{t} / 0.5 n_{2}\right)}{k}, \\
S_{t}=\sqrt{\frac{\sum_{j=1}^{n_{2}}\left(X_{t j}-\overline{X_{t}}\right)^{2}}{n_{2}-1}} \\
c_{4}=\left(\frac{2}{n_{2}-1}\right)^{0.5} \frac{\Gamma\left(0.5 n_{2}\right)}{\Gamma\left(0.5\left(n_{2}-1\right)\right)}, \quad \bar{S}=\frac{\sum_{t=1}^{k} S_{t}}{k} .
\end{gathered}
$$

The EWMA-AVand EWMA-AM charts are thus constructed using these estimated values of $p_{m 0}$ and $p_{v 0}$. The statistics $\mathrm{EWMA}_{M}$ and $\mathrm{EWMA}_{V}$ corresponding to the samples of sizes $n_{1}$ and $n_{2}$ are plotted on the resulting EWMA$\mathrm{AV}$ and EWMA-AM charts simultaneously. If no points fall outside their control limits, then we would deem the process to be in control.

\section{Example}

We will use an example from Yang et al. [17] to illustrate the new EWMA-AV and EWMA-AM charts. 
TABLE 13: (a) The new service times from the first ten counters in a bank branch $\left(\lambda_{1}=\lambda_{2}=0.05\right)$. (b) The new service times from the last ten counters in a bank branch $\left(\lambda_{1}=\lambda_{2}=0.05\right)$.

(a)

\begin{tabular}{cccccccccccccc}
\hline$t$ & $X_{1}$ & $X_{2}$ & $X_{3}$ & $X_{4}$ & $X_{5}$ & $X_{6}$ & $X_{7}$ & $X_{8}$ & $X_{9}$ & $X_{10}$ & $M_{t}$ & EWMA $_{M_{i}}$ \\
\hline 1 & 3.54 & 0.01 & 1.33 & 7.27 & 5.52 & 0.09 & 1.84 & 1.04 & 2.91 & 0.63 & 1 & 3.76 \\
2 & 0.86 & 1.61 & 1.15 & 0.96 & 0.54 & 3.05 & 4.11 & 0.63 & 2.37 & 0.05 & 0 & 3.57 \\
3 & 1.45 & 0.19 & 4.18 & 0.18 & 0.02 & 0.70 & 0.80 & 0.97 & 3.60 & 2.94 & 0 & 3.39 \\
4 & 1.37 & 0.14 & 1.54 & 1.58 & 0.45 & 6.01 & 4.59 & 1.74 & 3.92 & 4.82 & 1 & 3.27 \\
5 & 3.00 & 2.46 & 0.06 & 1.80 & 3.25 & 2.13 & 2.22 & 1.37 & 2.13 & 0.25 & 0 & 3.11 \\
6 & 1.59 & 3.88 & 0.39 & 0.54 & 1.58 & 1.70 & 0.68 & 1.25 & 6.83 & 0.31 & 1 & 3.00 \\
7 & 5.01 & 1.85 & 3.10 & 1.00 & 0.09 & 1.16 & 2.69 & 2.79 & 1.84 & 2.62 & 0 & 2.85 \\
8 & 4.96 & 0.55 & 1.43 & 4.12 & 4.06 & 1.42 & 1.43 & 0.86 & 0.67 & 0.13 & 0 & 2.71 \\
9 & 1.08 & 0.65 & 0.91 & 0.88 & 2.02 & 2.88 & 1.76 & 2.87 & 1.97 & 0.62 & 0 & 2.57 \\
10 & 4.56 & 0.44 & 5.61 & 2.79 & 1.73 & 2.46 & 0.53 & 1.73 & 7.02 & 2.13 & 1 & 2.49 \\
\hline
\end{tabular}

(b)

\begin{tabular}{lllllllllllll}
\hline$t$ & $X_{11}$ & $X_{12}$ & $X_{13}$ & $X_{14}$ & $X_{15}$ & $X_{16}$ & $X_{17}$ & $X_{18}$ & $X_{19}$ & $X_{20}$ & $V_{t}$ & EWMA $_{V_{i}}$ \\
\hline 1 & 5.01 & 3.88 & 4.18 & 0.88 & 4.06 & 3.05 & 4.59 & 0.63 & 6.83 & 0.25 & 0 & 1.14 \\
2 & 1.59 & 0.19 & 0.39 & 0.54 & 1.73 & 1.42 & 4.11 & 1.25 & 7.02 & 0.62 & 0 & 1.08 \\
3 & 1.59 & 0.65 & 1.43 & 1.00 & 5.52 & 1.16 & 4.11 & 1.37 & 3.6 & 2.62 & 0 & 1.03 \\
4 & 3.54 & 0.65 & 1.43 & 1.00 & 4.06 & 6.01 & 4.59 & 1.37 & 1.97 & 0.31 & 0 & 0.98 \\
5 & 5.01 & 0.01 & 1.54 & 7.27 & 0.09 & 2.88 & 4.11 & 0.97 & 7.02 & 0.63 & 0 & 0.93 \\
6 & 4.96 & 0.14 & 0.91 & 4.12 & 4.06 & 6.01 & 2.69 & 0.63 & 2.13 & 0.13 & 0 & 0.88 \\
7 & 4.96 & 2.46 & 1.54 & 7.27 & 0.45 & 1.42 & 0.53 & 0.63 & 2.13 & 4.82 & 0 & 0.84 \\
8 & 1.37 & 0.44 & 1.33 & 1.00 & 0.45 & 2.46 & 2.22 & 2.87 & 7.02 & 4.82 & 0 & 0.80 \\
9 & 1.59 & 0.01 & 0.91 & 1.58 & 0.54 & 1.70 & 1.84 & 2.79 & 2.91 & 0.62 & 0 & 0.76 \\
10 & 5.01 & 0.44 & 0.91 & 0.88 & 1.73 & 3.05 & 1.43 & 1.74 & 6.83 & 4.82 & 0 & 0.72 \\
\hline
\end{tabular}

Service time is an important quality characteristic in the banking industry. To measure the efficiency in the service system of a bank branch, the in-control sampling service times (unit: minutes) are measured from twenty counters every day for 15 days. That is, fifteen samples of size $n_{1}+n_{2}=$ 20 , where $n_{1}=n_{2}=10$, are available. This in-control data has been analyzed assuming a nonnormal distribution. For each sample, the first ten observations illustrated in Table 12(a) are used to calculate the $\mathrm{EWMA}_{M}$ statistic and the last ten observations illustrated in Table 12 (b) are used to calculate the $\mathrm{EWMA}_{V}$ statistic.

To construct the EWMA-AV and EWMA-AM charts, the variance and mean of the service time are estimated by $\left(\bar{S} / c_{4}\right)^{2}$ and $\overline{\bar{x}}$ using the fifteen samples in Tables $12(\mathrm{~b})$ and $12(\mathrm{a})$, respectively. The estimate of the variance is $\widehat{\sigma^{2}}=$ $\left(\bar{S} / c_{4}\right)^{2}=30.159$ and the estimate of the mean is $\widehat{\mu}=\overline{\bar{x}}=$ 5.77. For each sample in Table 12(b), the monitoring statistic EWMA $_{V}=$ total number of $\left(Y_{j}^{*}>30.159\right), j=1,2, \ldots, 10$, is calculated. For each sample in Table 12(a), the monitoring statistic $\mathrm{EWMA}_{M}=$ total number of $\left(Y_{j}>5.77\right), j=$ $1,2, \ldots, 10$, is calculated. Hence, the estimates of proportions $\left(p_{m 0}, p_{v 0}\right)$ are $\left(\widehat{p}_{m 0}=\left(\sum_{t=1}^{15} M_{t} / 10\right) / 15=0.39, \widehat{p}_{v 0}=\right.$ $\left.\left(\sum_{t=1}^{15} V_{t} / 5\right) / 15=0.24\right)$. The EWMA-AV and EWMA-AM charts with $\lambda_{1}=\lambda_{2}=0.05$ are constructed as follows based on the fifteen in-control samples, respectively.

The EWMA-AV chart:

$$
\mathrm{UCL}_{\text {EWMA- } V}=1.59, \quad \mathrm{LCL}_{\mathrm{EWMA}-V}=0.83 \text {. }
$$

The EWMA-AM chart:

$$
\mathrm{UCL}_{\mathrm{EWMA}-M}=4.51, \quad \mathrm{LCL}_{\mathrm{EWMA}-M}=3.28 \text {. }
$$

The monitoring statistics $\mathrm{EWMA}_{V}$ and $\mathrm{EWMA}_{M}$ are calculated (see Tables 12(b) and 12(a)). The EWMA-AV and EWMA-AM charts show no signals (see Figures 1(a) and 1(b)).

For comparison, we constructed the corresponding Shewhart mean and standard deviation $(S-\bar{X})$ charts and the transformed $S-\bar{X}$ charts by applying $X^{0.278}$ transformation because $X$ is a right-skewed distribution (see [20]). The Shewhart $S-\bar{X}$ charts, the transformed $S-\bar{X}$ charts, and the 
TABLE 14: (a) The service times from the first ten counters in a bank branch $\left(\lambda_{1}=\lambda_{2}=0.2\right)$. (b) The service times from the last ten counters in a bank branch $\left(\lambda_{1}=\lambda_{2}=0.2\right)$.

(a)

\begin{tabular}{cccccccccccccc}
\hline$t$ & $X_{1}$ & $X_{2}$ & $X_{3}$ & $X_{4}$ & $X_{5}$ & $X_{6}$ & $X_{7}$ & $X_{8}$ & $X_{9}$ & $X_{10}$ & $M_{t}$ & $\mathrm{EWMA}_{M_{i}}$ \\
\hline 1 & 0.88 & 0.78 & 5.06 & 5.45 & 2.93 & 6.11 & 11.59 & 1.20 & 0.89 & 3.21 & 2 & 3.52 \\
2 & 3.82 & 13.4 & 5.16 & 3.20 & 32.27 & 3.68 & 3.14 & 1.58 & 2.72 & 7.71 & 3 & 3.42 \\
3 & 1.40 & 3.89 & 10.88 & 30.85 & 0.54 & 8.40 & 5.10 & 2.63 & 9.17 & 3.94 & 4 & 3.53 \\
4 & 16.8 & 8.77 & 8.36 & 3.55 & 7.76 & 1.81 & 1.11 & 5.91 & 8.26 & 7.19 & 7 & 4.23 \\
5 & 0.24 & 9.57 & 0.66 & 1.15 & 2.34 & 0.57 & 8.94 & 5.54 & 11.69 & 6.58 & 4 & 4.18 \\
6 & 4.21 & 8.73 & 11.44 & 2.89 & 19.49 & 1.20 & 8.01 & 6.19 & 7.48 & 0.07 & 6 & 4.54 \\
7 & 15.08 & 7.43 & 4.31 & 6.14 & 10.37 & 2.33 & 1.97 & 1.08 & 4.27 & 14.08 & 5 & 4.64 \\
8 & 13.89 & 0.30 & 3.21 & 11.32 & 9.90 & 4.39 & 10.5 & 1.70 & 10.74 & 1.46 & 5 & 4.71 \\
9 & 0.03 & 12.76 & 2.41 & 7.41 & 1.67 & 3.70 & 4.31 & 2.45 & 3.57 & 3.33 & 2 & 4.17 \\
10 & 12.89 & 17.96 & 2.78 & 3.21 & 1.12 & 12.61 & 4.23 & 6.18 & 2.33 & 6.92 & 5 & 4.33 \\
11 & 7.71 & 1.05 & 1.11 & 0.22 & 3.53 & 0.81 & 0.41 & 3.73 & 0.08 & 2.55 & 1 & 3.67 \\
12 & 5.81 & 6.29 & 3.46 & 2.66 & 4.02 & 10.95 & 1.59 & 5.58 & 0.55 & 4.10 & 3 \\
13 & 2.89 & 1.61 & 1.30 & 2.58 & 18.65 & 10.77 & 18.23 & 3.13 & 3.38 & 6.34 & 4 & 3.53 \\
14 & 1.36 & 1.92 & 0.12 & 11.08 & 8.85 & 3.99 & 4.32 & 1.71 & 1.77 & 1.94 & 2 & 3.63 \\
15 & 21.52 & 0.63 & 8.54 & 3.37 & 6.94 & 3.44 & 3.37 & 6.37 & 1.28 & 12.83 & 5 & 3.30 \\
\hline
\end{tabular}

(b)

\begin{tabular}{cccccccccccccc}
\hline$t$ & $X_{11}$ & $X_{12}$ & $X_{13}$ & $X_{14}$ & $X_{15}$ & $X_{16}$ & $X_{17}$ & $X_{18}$ & $X_{19}$ & $X_{20}$ & $V_{t}$ & $\mathrm{EWMA}_{V_{i}}$ \\
\hline 1 & 3.82 & 6.29 & 10.88 & 30.85 & 9.9 & 3.99 & 1.59 & 1.71 & 8.26 & 4.1 & 1 \\
2 & 0.24 & 12.76 & 11.44 & 3.2 & 3.53 & 0.57 & 18.23 & 2.45 & 2.72 & 6.92 & 3 & 1.16 \\
3 & 3.82 & 7.43 & 0.12 & 3.37 & 1.12 & 12.61 & 1.59 & 1.08 & 0.89 & 0.07 & 1 & 1.42 \\
4 & 13.89 & 3.89 & 5.16 & 11.32 & 4.02 & 0.57 & 8.01 & 6.19 & 1.77 & 6.58 & 1 & 1.34 \\
5 & 5.81 & 12.76 & 2.41 & 1.15 & 3.53 & 0.81 & 11.59 & 5.91 & 4.27 & 3.33 & 0 & 1.07 \\
6 & 12.89 & 8.73 & 10.88 & 2.89 & 18.65 & 10.95 & 0.41 & 3.13 & 4.27 & 7.71 & 1 & 1.06 \\
7 & 2.89 & 0.63 & 0.12 & 0.22 & 4.02 & 10.95 & 8.01 & 1.08 & 10.74 & 4.1 & 0 & 0.85 \\
8 & 16.8 & 1.05 & 1.3 & 3.2 & 2.34 & 0.81 & 4.32 & 3.13 & 0.08 & 1.46 & 1 & 0.88 \\
9 & 4.21 & 17.96 & 5.06 & 0.22 & 4.02 & 3.99 & 8.01 & 5.91 & 0.55 & 3.33 & 1 & 0.90 \\
10 & 12.89 & 8.77 & 11.44 & 7.41 & 1.12 & 1.81 & 4.32 & 5.58 & 0.89 & 14.08 & 1 & 0.92 \\
11 & 0.88 & 8.77 & 5.06 & 3.55 & 8.85 & 10.95 & 18.23 & 5.54 & 2.33 & 6.58 & 2 \\
12 & 7.71 & 7.43 & 0.12 & 2.58 & 1.12 & 2.33 & 4.23 & 2.63 & 4.27 & 3.33 & 0 & 0.14 \\
13 & 7.71 & 9.57 & 0.12 & 30.85 & 7.76 & 1.81 & 3.14 & 1.71 & 2.72 & 14.08 & 2 & 1.13 \\
14 & 2.89 & 1.05 & 2.41 & 11.32 & 32.27 & 8.4 & 1.97 & 2.45 & 11.69 & 12.83 & 2 \\
15 & 1.36 & 0.63 & 3.46 & 11.32 & 0.54 & 10.95 & 4.23 & 2.45 & 2.33 & 6.34 & 2 & 1.30 & 1.44 \\
\hline
\end{tabular}

EWMA-S and EWMA- $\bar{X}$ charts are constructed with bounds as follows:

$$
\begin{aligned}
\mathrm{UCL}_{S}=8.80, & \mathrm{LCL}_{S}=1,455, \\
\mathrm{UCL}_{\bar{X}}=10.98, & \mathrm{LCL}_{\overline{\mathrm{X}}}=0.55, \\
\mathrm{UCL}_{T S}=0.732, & \mathrm{LCL}_{T S}=0.121, \\
\mathrm{UCL}_{T \bar{X}}=1.77, & \mathrm{LCL}_{T \bar{X}}=1.08, \\
\mathrm{UCL}_{\text {EWMA-S }}=7.495, & \mathrm{LCL}_{\text {EWMA-S }}=2.485 .
\end{aligned}
$$

The Shewhart $S-\bar{X}$ charts had two false signals (Samples 2 and 3 on the $S$ chart) (see Figures 2(a) and 2(b)), the transformed $S-\bar{X}$ charts had one signal (Sample 11 on the transformed $\bar{X}$ chart) (see Figures $3(\mathrm{a})$ and $3(\mathrm{~b})$ ), but the EWMA- $S$ and EWMA- $\bar{X}$ charts had no signals (see Figures 4(a) and 4(b)).

To illustrate the out-of-control detection ability of the service times from the new proposed EWMA-AV and EWMAAM charts for the new automatic service system of the bank branch, 10 new samples of size 20 and new samples 1-10 were collected and listed in Table 13(b) (the last 10 observations in each new sample) and Table 13(a) (the first 10 observations in each new sample).

Both the corresponding EWMA-AV and EWMA-AM charts, respectively, detected out-of-control variance signals 
TABLE 15: (a) The new service times from the first ten counters in a bank branch $\left(\lambda_{1}=\lambda_{2}=0.2\right)$. (b) The new service times from the last ten counters in a bank branch $\left(\lambda_{1}=\lambda_{2}=0.2\right)$.

(a)

\begin{tabular}{cccccccccccccc}
\hline$t$ & $X_{1}$ & $X_{2}$ & $X_{3}$ & $X_{4}$ & $X_{5}$ & $X_{6}$ & $X_{7}$ & $X_{8}$ & $X_{9}$ & $X_{10}$ & $M_{t}$ & EWMA $_{M_{i}}$ \\
\hline 1 & 3.54 & 0.01 & 1.33 & 7.27 & 5.52 & 0.09 & 1.84 & 1.04 & 2.91 & 0.63 & 1 & 3.11 \\
2 & 0.86 & 1.61 & 1.15 & 0.96 & 0.54 & 3.05 & 4.11 & 0.63 & 2.37 & 0.05 & 0 & 2.49 \\
3 & 1.45 & 0.19 & 4.18 & 0.18 & 0.02 & 0.70 & 0.80 & 0.97 & 3.60 & 2.94 & 0 & 1.99 \\
4 & 1.37 & 0.14 & 1.54 & 1.58 & 0.45 & 6.01 & 4.59 & 1.74 & 3.92 & 4.82 & 1 & 1.79 \\
5 & 3.00 & 2.46 & 0.06 & 1.80 & 3.25 & 2.13 & 2.22 & 1.37 & 2.13 & 0.25 & 0 & 1.43 \\
6 & 1.59 & 3.88 & 0.39 & 0.54 & 1.58 & 1.70 & 0.68 & 1.25 & 6.83 & 0.31 & 1 & 1.35 \\
7 & 5.01 & 1.85 & 3.10 & 1.00 & 0.09 & 1.16 & 2.69 & 2.79 & 1.84 & 2.62 & 0 & 1.08 \\
8 & 4.96 & 0.55 & 1.43 & 4.12 & 4.06 & 1.42 & 1.43 & 0.86 & 0.67 & 0.13 & 0 & 0.86 \\
9 & 1.08 & 0.65 & 0.91 & 0.88 & 2.02 & 2.88 & 1.76 & 2.87 & 1.97 & 0.62 & 0 & 0.69 \\
10 & 4.56 & 0.44 & 5.61 & 2.79 & 1.73 & 2.46 & 0.53 & 1.73 & 7.02 & 2.13 & 1 & 0.75 \\
\hline
\end{tabular}

(b)

\begin{tabular}{lllllllllllll}
\hline$t$ & $X_{11}$ & $X_{12}$ & $X_{13}$ & $X_{14}$ & $X_{15}$ & $X_{16}$ & $X_{17}$ & $X_{18}$ & $X_{19}$ & $X_{20}$ & $V_{t}$ & $\mathrm{EWMA}_{V_{i}}$ \\
\hline 1 & 5.01 & 3.88 & 4.18 & 0.88 & 4.06 & 3.05 & 4.59 & 0.63 & 6.83 & 0.25 & 0 & 1.15 \\
2 & 1.59 & 0.19 & 0.39 & 0.54 & 1.73 & 1.42 & 4.11 & 1.25 & 7.02 & 0.62 & 0 & 0.92 \\
3 & 1.59 & 0.65 & 1.43 & 1.00 & 5.52 & 1.16 & 4.11 & 1.37 & 3.6 & 2.62 & 0 & 0.74 \\
4 & 3.54 & 0.65 & 1.43 & 1.00 & 4.06 & 6.01 & 4.59 & 1.37 & 1.97 & 0.31 & 0 & 0.59 \\
5 & 5.01 & 0.01 & 1.54 & 7.27 & 0.09 & 2.88 & 4.11 & 0.97 & 7.02 & 0.63 & 0 & 0.47 \\
6 & 4.96 & 0.14 & 0.91 & 4.12 & 4.06 & 6.01 & 2.69 & 0.63 & 2.13 & 0.13 & 0 & 0.38 \\
7 & 4.96 & 2.46 & 1.54 & 7.27 & 0.45 & 1.42 & 0.53 & 0.63 & 2.13 & 4.82 & 0 & 0.30 \\
8 & 1.37 & 0.44 & 1.33 & 1.00 & 0.45 & 2.46 & 2.22 & 2.87 & 7.02 & 4.82 & 0 & 0.24 \\
9 & 1.59 & 0.01 & 0.91 & 1.58 & 0.54 & 1.70 & 1.84 & 2.79 & 2.91 & 0.62 & 0 & 0.19 \\
10 & 5.01 & 0.44 & 0.91 & 0.88 & 1.73 & 3.05 & 1.43 & 1.74 & 6.83 & 4.82 & 0 & 0.15 \\
\hline
\end{tabular}

EWMA-AV chart

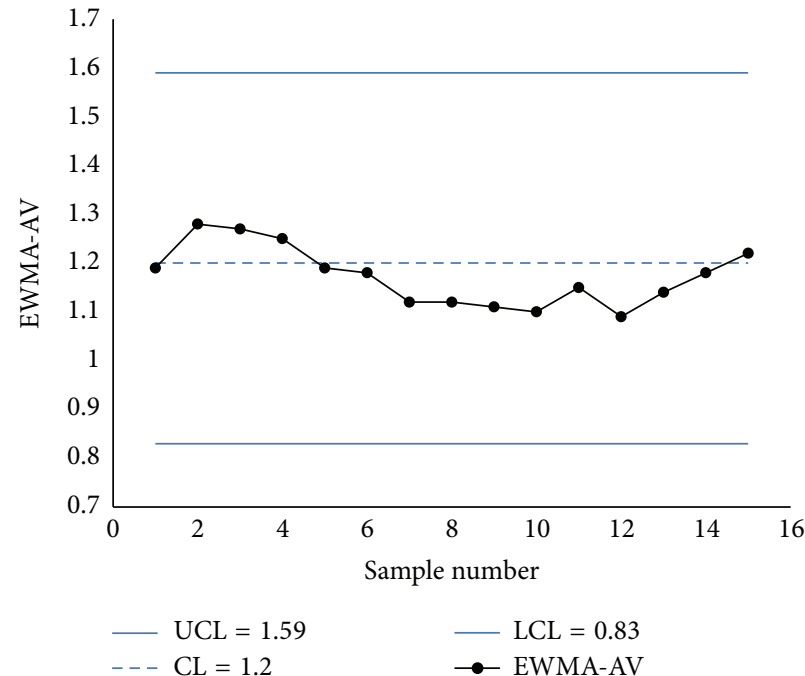

(a)

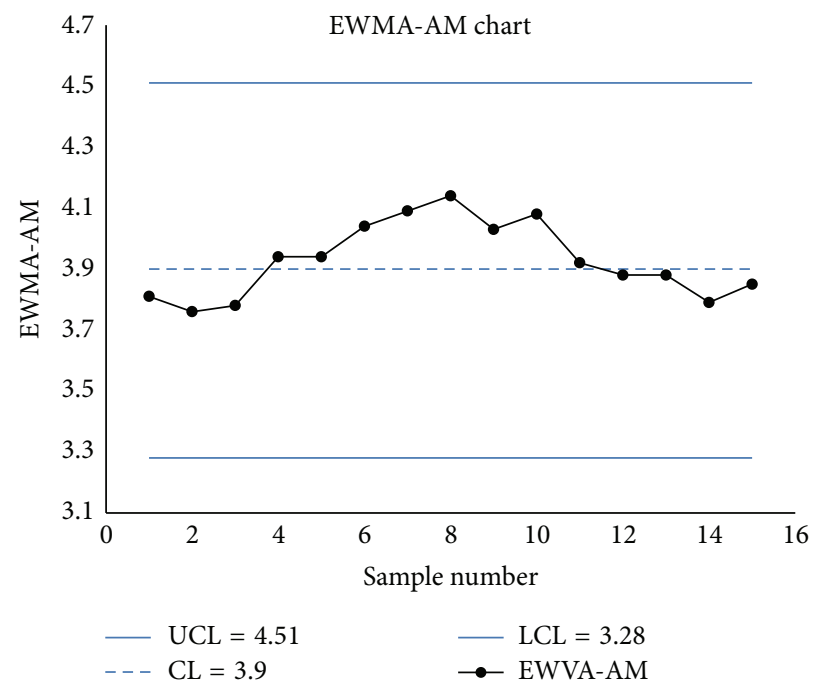

(b)

Figure 1: (a) The EWMA-AV chart. (b) The EWMA-AM chart. 


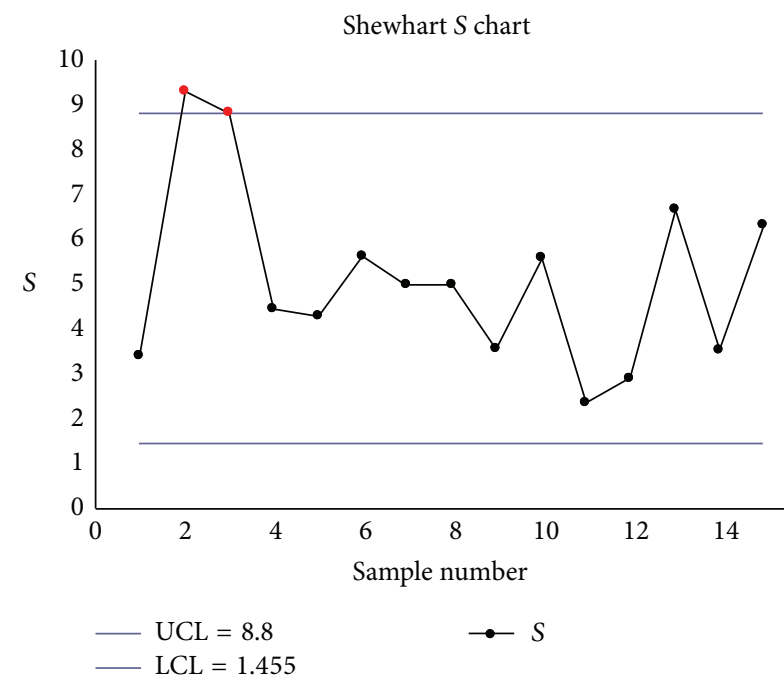

(a)

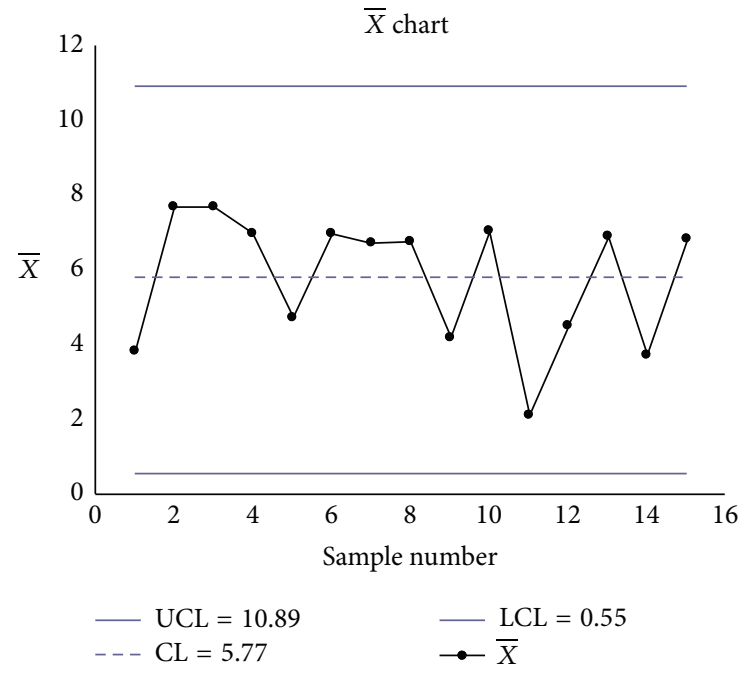

(b)

FIgure 2: (a) Shewhart $S$ chart. (b) Shewhart $\bar{X}$ chart.

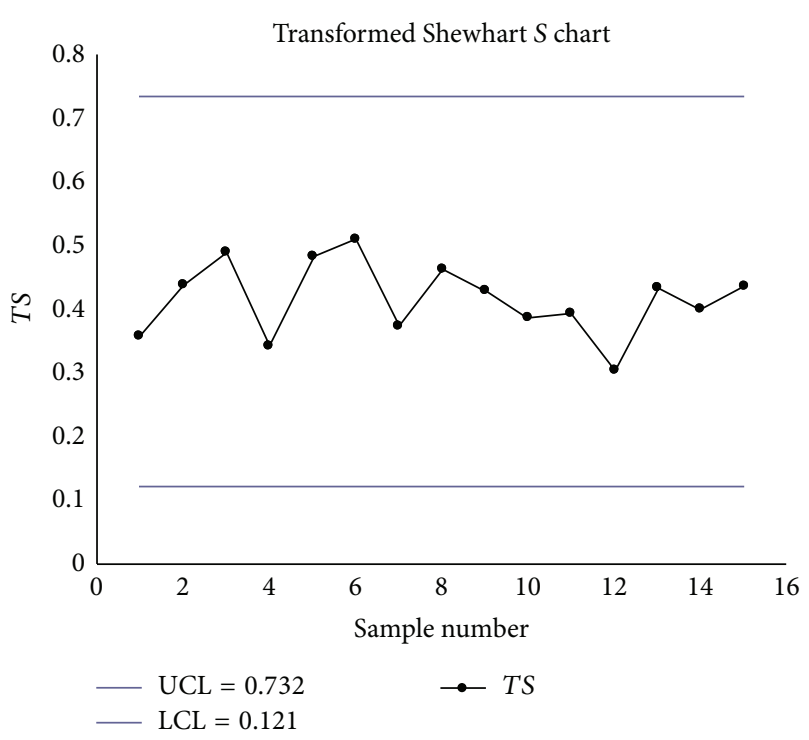

(a)

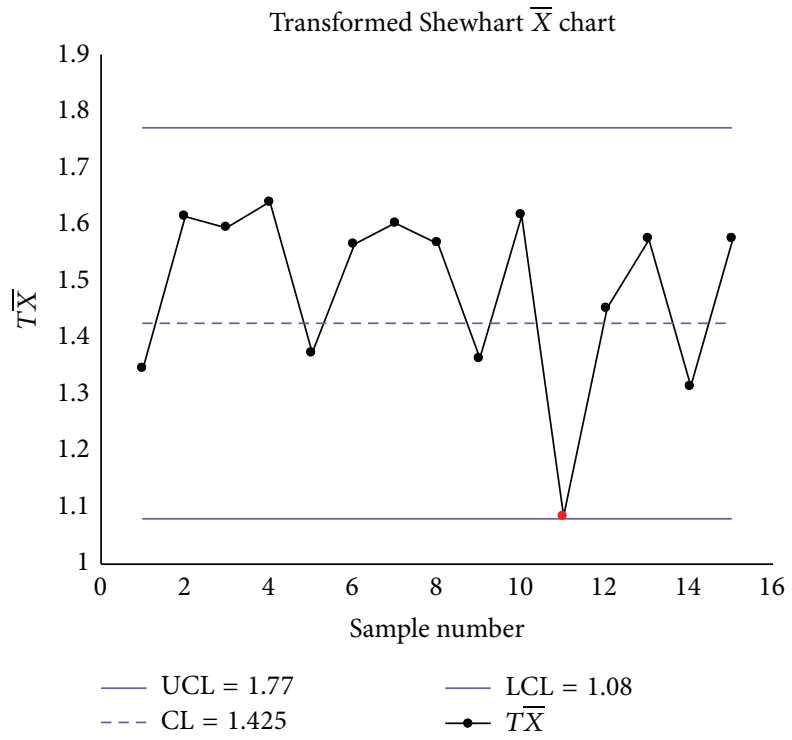

(b)

Figure 3: (a) The transformed Shewhart $S$ chart. (b) The transformed $\bar{X}$ chart.

from the eighth sample onward and the out-of-control mean signal from the fourth sample onward (samples 8-10 on EWMA-AV chart and samples 4-10 EWMA-AM chart) (see Figures 5(a) and 5(b)). That is, the variance and mean of the new service times are significantly reduced because of the improved new automatic service system. However, the corresponding Shewhart $S-\bar{X}$ charts produced only four true out-of-control standard deviation signals (samples 2, 5, 7, and 9 on $S$ chart) (see Figures 6(a) and 6(b)), the transformed Shewhart $S-\bar{X}$ charts produced only two out-of-control mean signals (samples 2 and 3 on transformed $\bar{X}$ chart) (see Figures $7(a)$ and $7(b))$. Both the corresponding EWMA-S and
EWMA- $\bar{X}$ charts detected only out-of-control mean signals from the fourth sample onward (samples 4-10 on EWMA- $\bar{X}$ chart) (see Figures 8(a) and 8(b)).

Construction of the Shewhart $S-\bar{X}$ charts, the transformed $S-\bar{X}$ charts, and the EWMA-S and EWMA- $\bar{X}$ charts requires a normality assumption but this is not the case for the proposed EWMA-AV and EWMA-AM charts. In this example, neither the $S-\bar{X}$ charts nor the transformed $S-\bar{X}$ charts detected most of the out-of-control signals. The new EWMAAV and EWMA-AM charts showed superior detection ability than the existing charts in monitoring and detecting process variance and mean shifts. As a consequence, in the final 

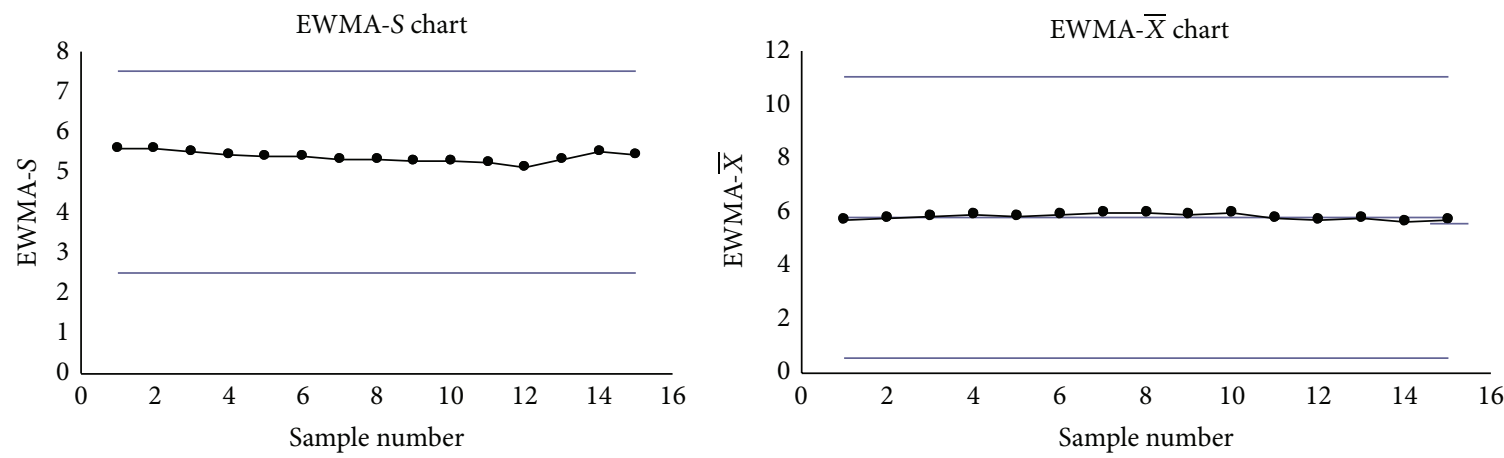

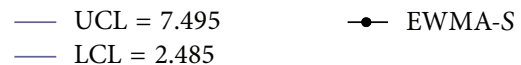

(a)

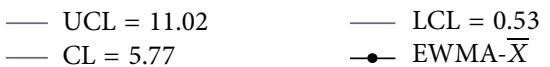

(b)

Figure 4: (a) The EWMA ${ }_{S}$ chart. (b) The EWMA $\overline{\bar{X}}$ chart.

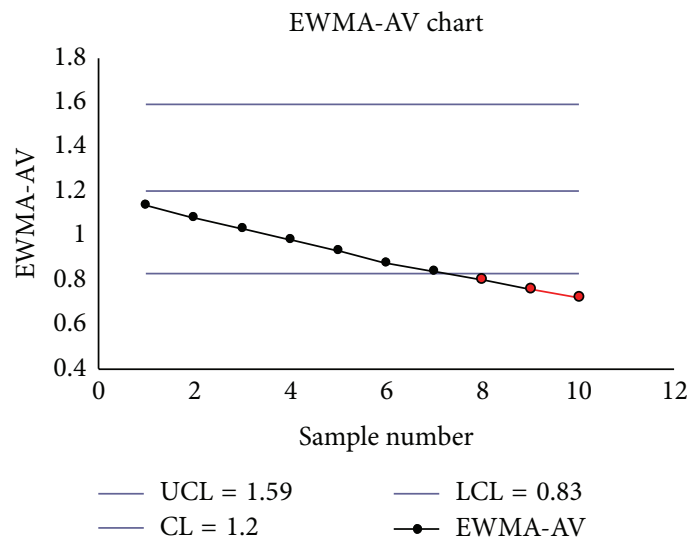

(a)

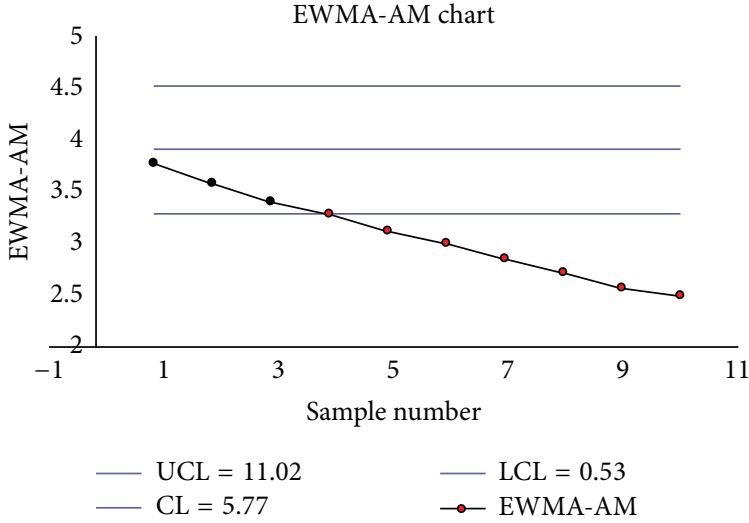

(b)

Figure 5: (a) The EWMA-AV chart. (b) The EWMA-AM chart.

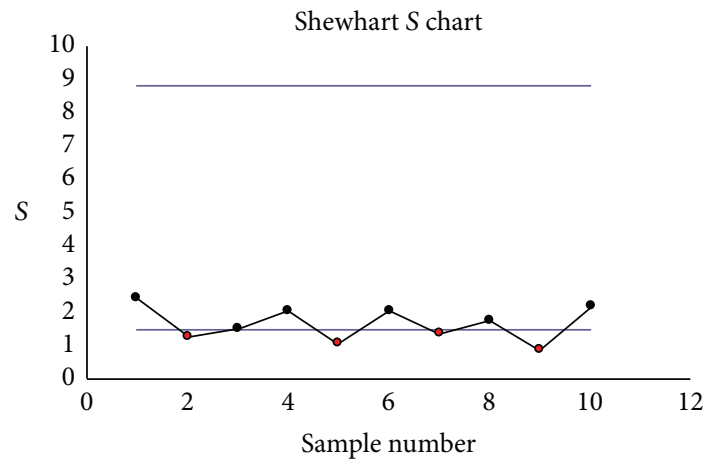

$\begin{aligned} \mathrm{UCL} & =8.8 \\ \mathrm{LCL} & =1.455\end{aligned} \rightarrow \mathrm{S}$

(a)

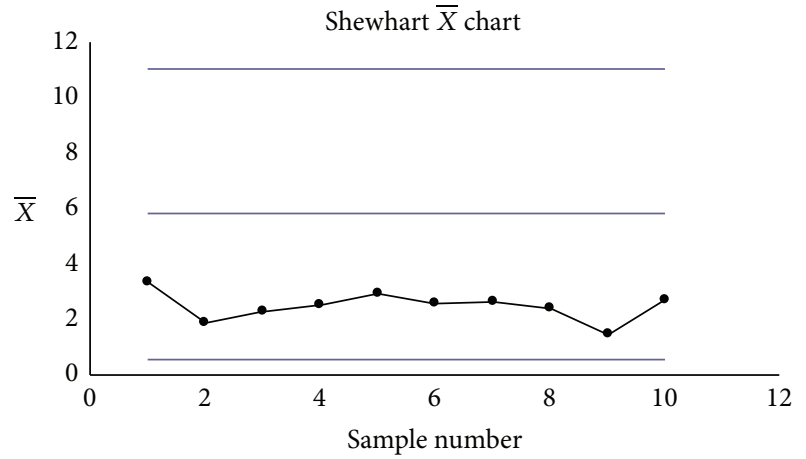

$\longrightarrow \underset{\mathrm{X}}{\mathrm{LCL}}=0.55$

(b)

Figure 6: (a) The Shewhart $S$ chart. (b) The Shewhart $\bar{X}$ chart. 


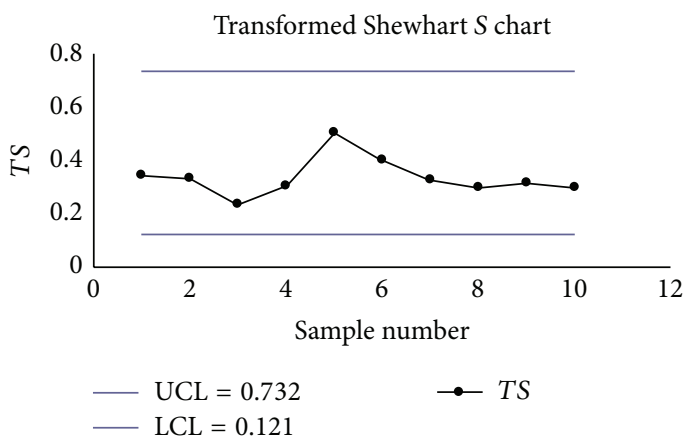

(a)

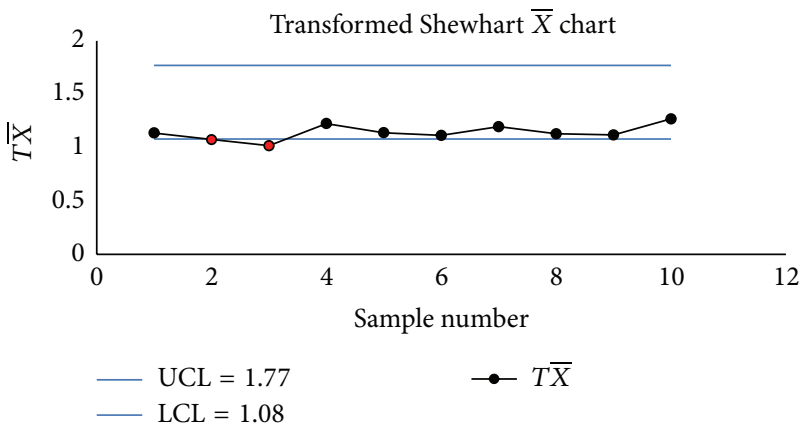

(b)

FIgURE 7: (a) The transformed Shewhart $S$ chart. (b) The transformed Shewhart $\bar{X}$ chart.

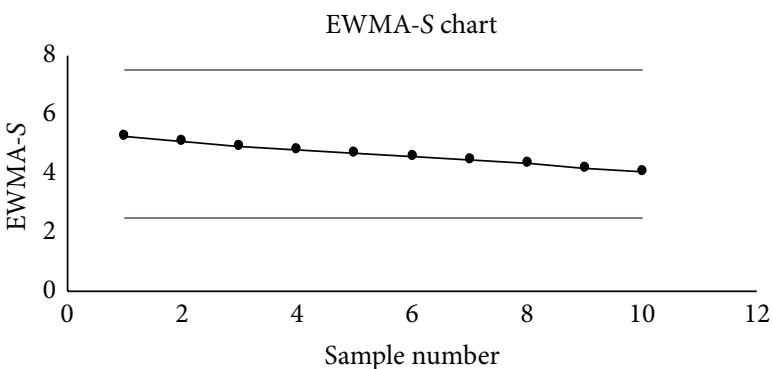

$\mathrm{UCL}=7.495$
$\mathrm{LCL}=2.485$

(a)
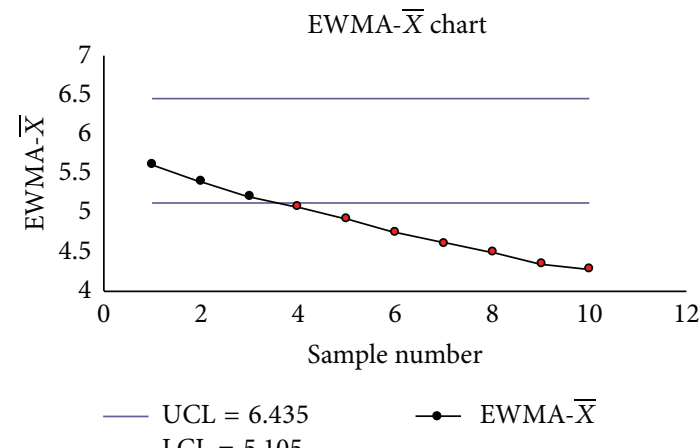

(b)

Figure 8: (a) The EWMA ${ }_{S}$ chart. (b) The EWMA $\overline{\bar{X}}$ chart.

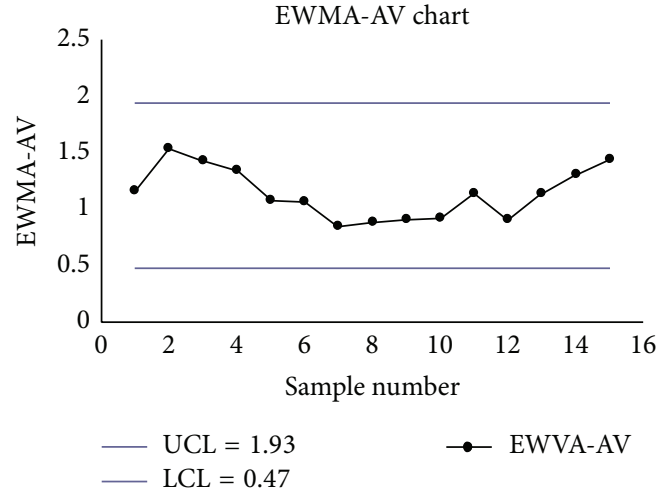

(a)

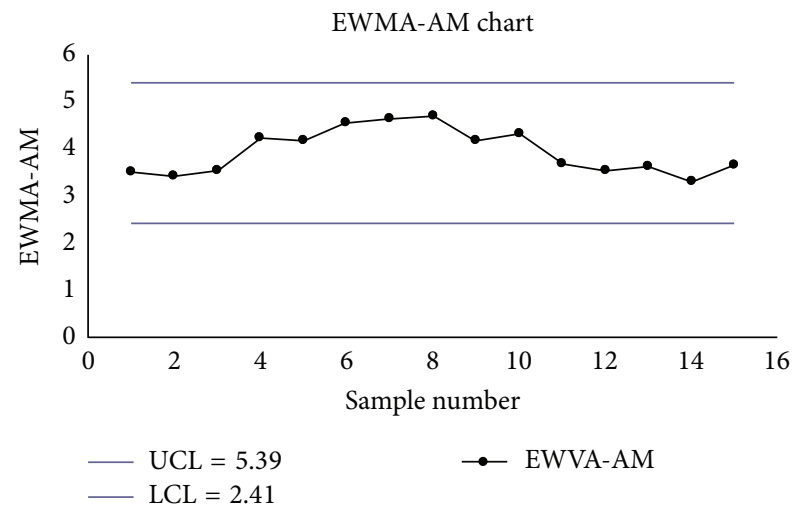

(b)

Figure 9: (a) The EWMA-AV chart with $\lambda_{2}=0.2$. (b) The EWMA-AM chart with $\lambda_{1}=0.2$.

analysis, the EWMA-AV and EWMA-AM charts appear to be the best.

Alternatively, if we take $\lambda_{1}=\lambda_{2}=0.2$ then the EWMAAV and EWMA-AM charts are as follows based on the fifteen in-control samples in Tables 12(b) and 12(a).

The EWMA-AV chart:

$$
\mathrm{UCL}_{\text {EWMA- } V}=1.93, \quad \mathrm{LCL}_{\mathrm{EWMA}-V}=0.47 \text {. }
$$

The EWMA-AM chart:

$$
\mathrm{UCL}_{\text {EWMA-M }}=5.39, \quad \mathrm{LCL}_{\mathrm{EWMA}-M}=2.41 \text {. }
$$

The monitoring in-control statistics $\mathrm{EWMA}_{M}$ and EWMA $_{V}$ are listed in Tables 14(b) and 14(a). The EWMA-AV and EWMA-AM charts show no signals (see Figures 9(a) and $9(\mathrm{~b})$ ). 


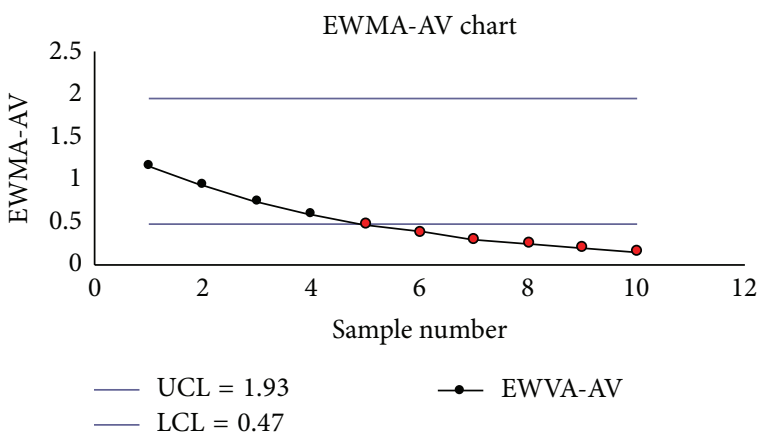

(a)

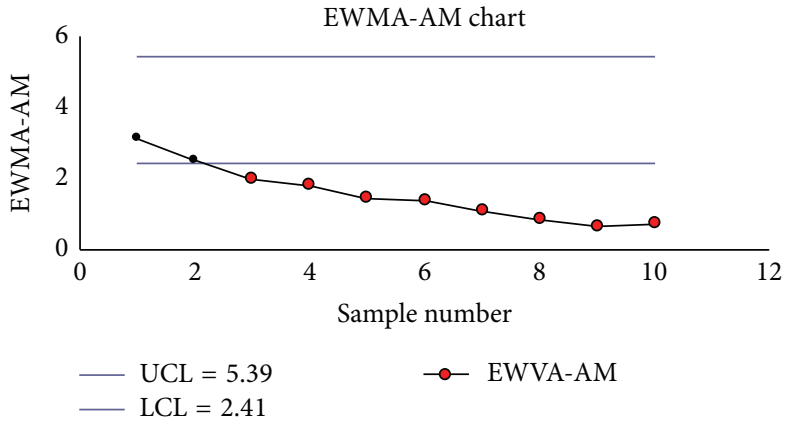

(b)

FIgure 10: (a) The EWMA-AV chart with $\lambda_{2}=0.2$. (b) The EWMA-AM chart with $\lambda_{1}=0.2$.

The monitoring statistics of the EWMA-AM and EWMA$\mathrm{AV}$ charts for the new automatic service system of the bank branch were listed in Tables 15(a) and 15(b).

Both the corresponding EWMA-AV and EWMA-AM charts, respectively, detected out-of-control variance signals from the fifth sample onward and the out-of-control mean signal from the third sample onward (samples 5-10 on EWMA-AV chart and samples 3-10 EWMA-AM charts) (see Figures 10(a) and 10(b)). That is, the EWMA-AV and EWMAAM charts with $\lambda_{1}=\lambda_{2}=0.2$ detected the out-of-control variance and mean earlier than that of the EWMA-AV and EWMA-AM charts with $\lambda_{1}=\lambda_{2}=0.05$. The reason is that the mean and variance had larger shifts; that is, the $\widehat{p}_{m o}=0.39$ changed to $\widehat{p}_{m 1}=0.04$ and $\widehat{p}_{v o}=0.24$ changed to $\widehat{p}_{v 1}=0.00$. A good rule of thumb for using EWMA control chart is to use a larger weight, $\lambda$, to detect larger shift (see [20]).

\section{Conclusions}

In this paper, we propose using both the new EWMA-AM and EWMA-AV charts, based on two simple independent statistics to monitor the variance and mean shifts in the process simultaneously when the distribution of a quality characteristic is not known or is not believed to be normal. The EWMA-AM and EWMA-AV charts improve the detection ability of the symmetric mean chart and symmetric variance chart constructed using binomial distributions. Furthermore, the new EWMA-AM and EWMA-AV charts provide more intuitive and reasonable in-control and outof-control average run lengths. A numerical example of service times from a bank branch with a right skewed distribution illustrated the application of the new EWMAAM and EWMA-AV charts which were compared with some existing charts. The new EWMA-AM and EWMA-AV charts showed superior detection ability than the existing charts in monitoring and detecting both the process mean and variance shifts. The new EWMA-AM and EWMA-AV charts are thus recommended.

\section{Conflict of Interests}

The authors declare that there is no conflict of interests regarding the publication of this paper.

\section{Acknowledgments}

The research was partially supported by NSC 100-2118M-004-003-MY2 Research Grant, Taiwan, the Commercial College of National Chengchi University, Taiwan, and NCTS, Taiwan.

\section{References}

[1] B. L. MacCarthy and T. Wasusri, "A review of non-standard applications of statistical rocess control (SPC) charts," International Journal of Quality and Reliability Management, vol. 19, no. 3, pp. 295-320, 2002.

[2] F. Tsung, Y. Li, and M. Jin, "Statistical process control for multistage manufacturing and service operations: a review and some extensions," International Journal of Services Operations and Informatics, vol. 3, no. 2, pp. 191-204, 2008.

[3] X. Ning, Y. Shang, and F. Tsung, "Statistical process control techniques for service processes: a review," in Proceedings of the 6th International Conference on Service Systems and Service Management (ICSSSM '09), pp. 927-931, Xiamen, China, June 2009.

[4] E. B. Ferrell, "Control charts using midranges and medians," Industrial Quality Control, vol. 9, pp. 30-34, 1953.

[5] S. T. Bakir and M. R. Reynolds Jr., "A nonparametric procedure for process control based on within group ranking," Technometrics, vol. 21, no. 2, pp. 175-183, 1979.

[6] R. Amin, M. R. Reynolds Jr., and S. Baker, "Nonparametric quality control charts based on the sign statistic," Communications in Statistics-Theory and Methods, vol. 24, pp. 1597-1624, 1995.

[7] S. Chakraborti, P. Lann, and M. A. Van der Wiel, "Nonparametric control charts: an overview and some results," Journal of Quality Technology, vol. 33, no. 3, pp. 304-315, 2001.

[8] P. F. Altukife, "A new nonparametric control charts based on the observations exceeding the grand median," Pakistan Journal of Statistics, vol. 19, no. 3, pp. 343-351, 2003.

[9] F. Altukife, "Nonparametric control charts based on sum of ranks," Pakistan Journal of Statistics, vol. 19, no. 3, pp. 291-300, 2003.

[10] S. T. Bakir, "A distribution-free Shewhart quality control chart based on signed-ranks," Quality Engineering, vol. 16, no. 4, pp. 613-623, 2004.

[11] S. Chakraborti and S. Eryilmaz, "A nonparametric shewharttype signed-rank control chart based on runs," Communications 
in Statistics-Simulation and Computation, vol. 36, no. 2, pp. 335-356, 2007.

[12] S. Chakraborti and M. Graham, Nonparametric Control Charts, Encyclopedia of Quality and Reliability, John Wiley \& Sons, New York, NY, USA, 2007.

[13] S. Chakraborti and M. A. van der Wiel, "A nonparametric control chart based on the Mann-Whitney Statistic," in Beyond Parametrics in Interdisciplinary Research: Festschrift in Honor of Professor Pranab K. Sen, pp. 156-172, Institute of Mathematical Statistics, Beachwood, Ohio, USA, 2008.

[14] S. Li, L. Tang, and S. Ng, "Nonparametric CUSUM and EWMA control charts for detecting mean shifts," Journal of Quality Technology, vol. 42, no. 2, pp. 209-226, 2010.

[15] C. Zou and F. Tsung, "Likelihood ratio-based distribution-free EWMA control charts," Journal of Quality Technology, vol. 42, no. 2, pp. 174-196, 2010.

[16] N. Das and A. Bhattacharya, "A new non-parametric control chart for controlling variability," Quality Technology and Quantitative Management, vol. 5, no. 4, pp. 351-361, 2008.

[17] S. F. Yang, T. Cheng, Y. Hung, and S. Cheng, "A new chart for monitoring service process mean," Quality and Reliability Engineering International, vol. 28, no. 4, pp. 377-386, 2012.

[18] S. F. Yang and S. W. Cheng, "A new non-parametric CUSUM mean chart," Quality and Reliability Engineering International, vol. 27, no. 7, pp. 867-875, 2011.

[19] S. F. Yang, J.-S. Lin, and S. W. Cheng, "A new nonparametric EWMA sign control chart," Expert Systems with Applications, vol. 38, no. 5, pp. 6239-6243, 2011.

[20] D. C. Montgomery, Introduction to Statistical Quality Control, Wiley, New York, NY, USA, 2009.

[21] J. M. Lucas and M. S. Saccucci, "Exponentially weighted moving average control schemes. Properties and enhancements," Technometrics, vol. 32, no. 1, pp. 1-12, 1990.

[22] S. F. Yang, "Using a new VSI EWMA average loss control chart to monitor changes in the difference between the process mean and target and/or the process variability," Applied Mathematical Modeling, vol. 37, no. 16-17, pp. 7973-7982, 2013. 


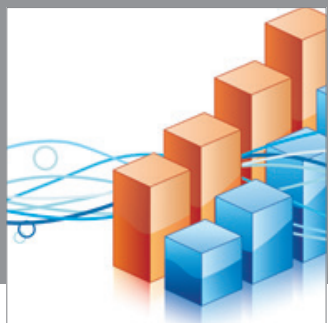

Advances in

Operations Research

mansans

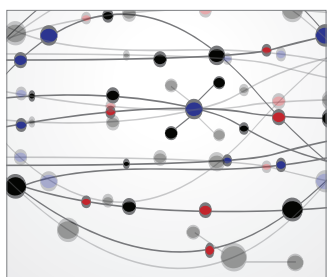

The Scientific World Journal
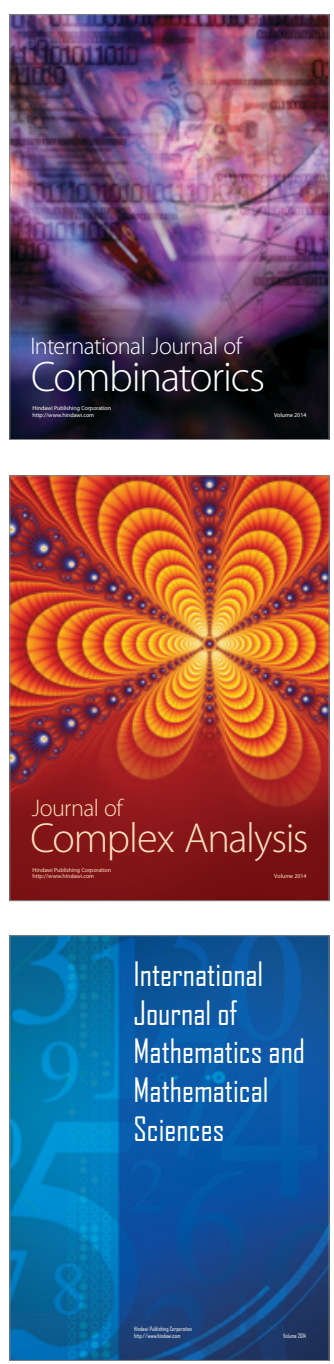
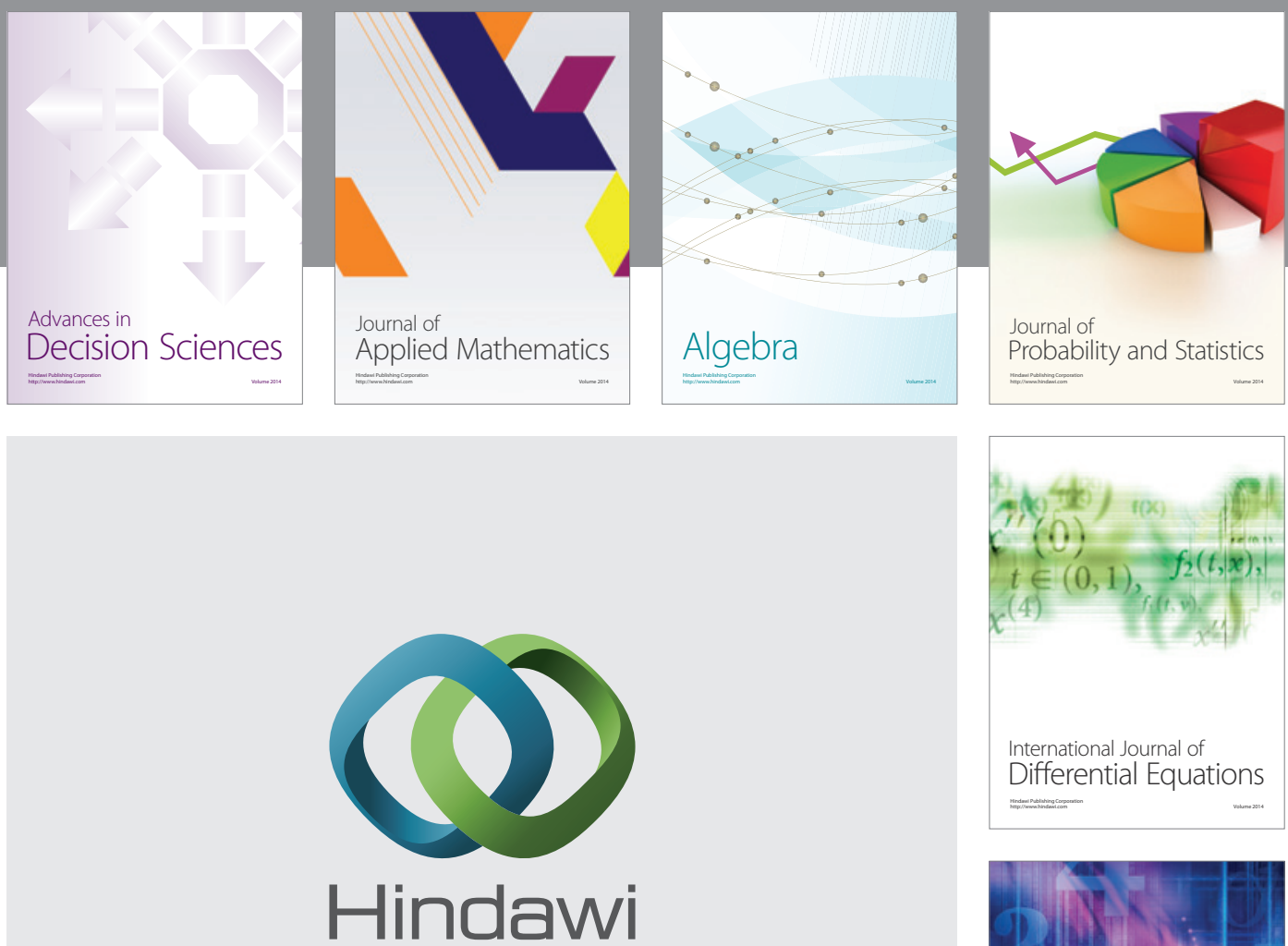

Submit your manuscripts at http://www.hindawi.com
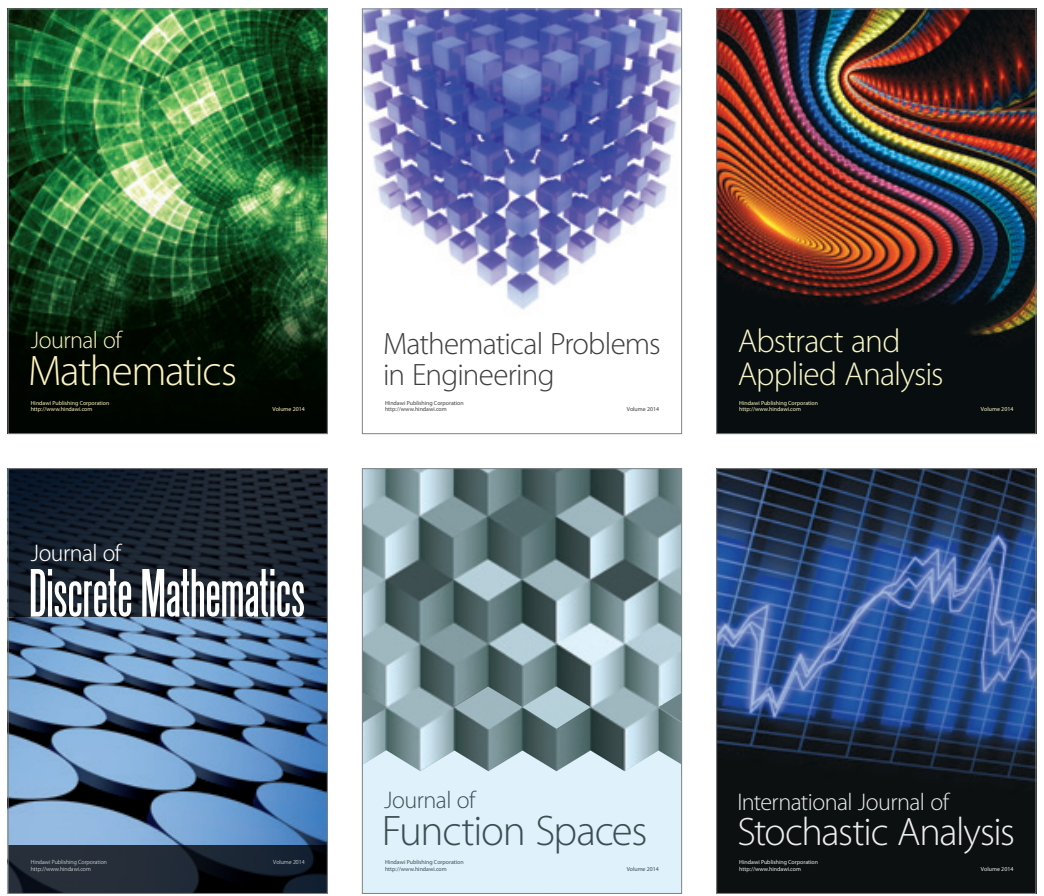

Journal of

Function Spaces

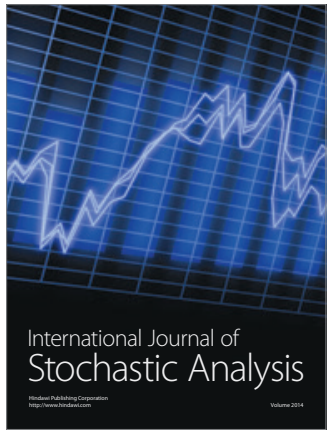

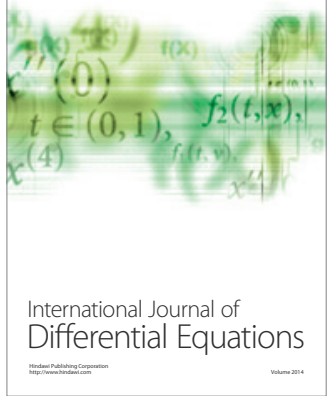
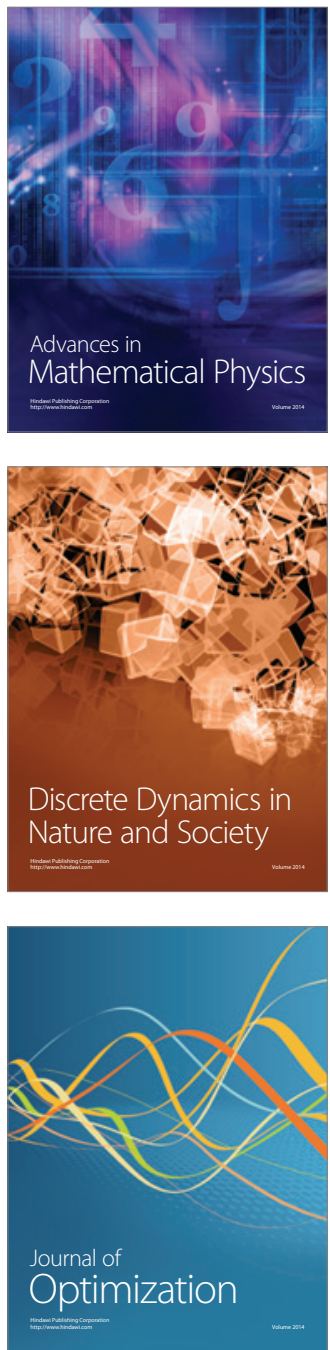